Accepted for publication in Math. Struct. in Comp. Science

\title{
Borel and Hausdorff Hierarchies in Topological Spaces of Choquet Games and Their Effectivization
}

\author{
Verónica Becher ${ }^{1 \dagger}$ and Serge Grigorieff ${ }^{2 \dagger}$ \\ ${ }^{1}$ FCEyN, Universidad de Buenos Aires \& CONICET, Argentina. \\ ${ }^{2}$ LIAFA, CNRS $\&$ Université Paris Diderot - Paris 7, France.
}

Received August 21, 2012

\begin{abstract}
What parts of classical descriptive set theory done in Polish spaces still hold for more general topological spaces, possibly $T_{0}$ or $T_{1}$, but not $T_{2}$ (i.e. not Hausdorff)? This question has been addressed by Victor Selivanov in a series of papers centered on algebraic domains. And recently it has been considered by Matthew de Brecht for quasi-Polish spaces, a framework that contains both countably based continuous domains and Polish spaces. In this paper we present alternative unifying topological spaces, that we call approximation spaces. They are exactly the spaces for which player Nonempty has a stationary strategy in the Choquet game. A natural proper subclass of approximation spaces coincides with the class of quasi-Polish spaces. We study the Borel and Hausdorff difference hierarchies in approximation spaces, revisiting the work done for the other topological spaces. We also consider the problem of effectivization of these results.
\end{abstract}

\section{Introduction}

The primary setting of descriptive set theory, including the study of Borel and Hausdorff hierarchies, is that of Polish spaces. These are spaces homeomorphic to complete metric spaces that have a countable dense subset, for example the Cantor space, the Baire space, the real line and its intervals. The question of what parts of classical descriptive set theory still hold for non-Polish spaces, specifically for general $T_{0}$ topological spaces, has not been yet systematically studied. Major progress has been done by Victor Selivanov in his investigations centered mainly in algebraic domains (directed complete partial orders with a countable base of compact elements) in an ongoing series of papers on this topic that started in 1978. Recently, Matthew de Brecht (2011) presented the theory of quasi-Polish spaces, a unifying framework for Polish spaces and countably based domains (i.e., $\omega$-continuous domains, or directed complete partial orders with a countable basis).

\footnotetext{
$\dagger$ Members of the Laboratoire International Associé INFINIS, Universidad de Buenos Aires - Université Paris Diderot-Paris 7. This research was partially done whilst the first author was a visiting fellow at the Isaac Newton Institute for Mathematical Sciences in the programme 'Semantics \& Syntax'.
} 
De Brecht characterized quasi-Polish spaces in terms of the Choquet topological games, and he proved that descriptive set theory can be nicely developed in such spaces.

In this paper we consider alternative unifying topological spaces, that we call approximation spaces. Not only they contain all Polish spaces and all continuous domains, but a natural subclass of approximation spaces coincides with the class of quasi-Polish spaces. Approximation spaces can be viewed as the "à la domain" version of the "à la Polish" unifying framework of de Brecht. These spaces can also be characterized in terms of Choquet games. We study the Borel and Hausdorff difference hierarchies in approximation spaces, revisiting the work done for the other topological spaces. We also consider the problem of effectivization of these results.

The paper is organized as follows. 2 presents the preliminary material. We recall the needed notions about the Borel and Hausdorff hierarchies in a $T_{0}$ (possibly not $T_{2}$ ) topological context. We give an overview of the needed material on domains with the Scott topology and quasi-Polish spaces. We also present some prerequisites on the Banach-Mazur and Choquet topological games.

$\$ 3$ is devoted to the class of approximation spaces. We prove that both, Polish spaces and continuous domains, are approximation spaces. Indeed, we show that all quasi-Polish spaces are approximation spaces. Theorem 3.10 characterizes approximation spaces in terms of Choquet games. Theorem 3.12 proves that quasi-Polish spaces and convergent approximation spaces are the same class.

In the context of Polish spaces, the Baire property asserts that any countable intersection of dense open sets is dense. Thus, countable intersections of open sets, the $\mathbf{G}_{\delta}$ sets, constitute the $\Pi_{2}^{0}$ level of the Borel hierarchy. In the context of $T_{0}$ but not $T_{2}$ spaces this is not true any more: the $\boldsymbol{\Pi}_{2}^{0}$ level consists of countable intersections of Boolean combinations of open sets. Then it is natural to consider the $\boldsymbol{\Pi}_{2}^{0}$ Baire property which asserts that any countable intersection of dense differences of open sets is dense. As shown by de Brecht (2011), the usual $\mathbf{G}_{\delta}$ Baire property and Hausdorff-Kuratowski's theorem both hold for quasi-Polish spaces. Consequently, these two results are ensured for convergent approximation spaces. Theorem 3.14 proves that, in fact, all approximation spaces satisfy the $\Pi_{2}^{0}$ Baire property. Theorem 3.16 extends Hausdorff's theorem to spaces having a countable basis and such that every closed subspace is an approximation space: the $\boldsymbol{\Delta}_{2}^{0}$ class coincides with the difference hierarchy. This result was previously obtained by Selivanov for $\omega$-algebraic domains and then for $\omega$-continuous domains (Selivanov 2005, 2008). De Brecht (2011) proved that the full Hausdorff-Kuratowski Theorem holds for quasi-Polish spaces; hence, it holds for convergent approximation spaces. We do not know whether it holds for all approximation spaces.

In 84 we revisit a part of Selivanov's work $(2005,2008)$ on domains that does not apply to Polish spaces: his characterization of the classes of the Hausdorff hierarchy in terms of alternating trees, and his proof of non existence of ambiguous sets in this hierarchy. We check that the assumption of $\omega$-algebraicity or $\omega$-continuity can be replaced, mutatis mutandis, by that of continuity.

$\$ 5$ is devoted to effectivization. The definition of approximation spaces admits a straightforward definition of an effective version. We make the first steps in developing the effective theory. We first recall the notions of effective topological space and effective domains. 
We also include the known machinery of effective Borel codes. Thorem 5.17proves a weak effective version of Hausdorff's theorem in effective approximation spaces. We obtain this proof as an adaptation of Selivanov's work (2003) for the Baire space. A general effective version of Hausdorff's theorem is still an open question.

Acknowledgement. We thank Martín Escardó for pointing out the work of Matthew de Brecht on quasi-Polish spaces and for giving us very helpful references. Also we are indebted to an anonymous reviewer for his/her insights on approximation spaces; specifically, we owe this reviewer the indication to consider Choquet games and the convergence condition on approximation spaces that yields the equivalence with quasi-Polish spaces.

\section{Preliminary Definitions and Results}

We write $\mathbb{N}$ for the set of natural numbers, $\mathcal{P}(\mathbb{N})$ for the set of all subsets of $\mathbb{N}$ and $\mathcal{P}_{<\omega}(\mathbb{N})$ for the set of all finite subsets of $\mathbb{N}$. Finite sequences of elements of a set $X$ are denoted by $\left(x_{1}, x_{2}, \ldots, x_{n}\right)$. Concatenation of sequences $u, v$ and element $x$ are written simply as $u v, u x$. We use Greek letters to denote ordinals. We write $\omega$ for the first infinite ordinal, $\omega_{1}$ for the first uncountable ordinal, and $\omega_{1}^{C K}$ for the least not computable ordinal (the Church-Kleene ordinal). For any two ordinals $\alpha, \beta, \alpha \sim \beta$ means that they have the same parity.

\subsection{Borel and Hausdorff Hierarchies in General Topological Spaces}

All the material of this subsection on the Borel and Hausdorff hierarchies in general topological spaces has first appeared in (Selivanov 2005). To make the presentation self contained, we reproduce here some of the proofs.

2.1.1. The Borel Hierarchy. In general topological spaces, an open set may possibly not be a countable union of closed sets, cf. Remark 2.13 infra. In order to get the expected inclusion $\boldsymbol{\Sigma}_{1}^{0}(E) \subseteq \boldsymbol{\Sigma}_{2}^{0}(E)$, one has to distort the usual definition of Borel spaces given in metric spaces. This leads to define the hierarchy of Borel sets in a general setting as follows.

Definition 2.1 (Borel sets). Let $E$ be a topological space.

1 Borel subsets of $E$ are those sets obtained from open sets by iterated complementation and countable unions and intersections.

2 The Borel classes $\boldsymbol{\Sigma}_{\alpha}^{0}(E), \boldsymbol{\Pi}_{\alpha}^{0}(E), \boldsymbol{\Delta}_{\alpha}^{0}(E)$, where $\alpha \geq 1$ varies over countable ordinals, are inductively defined as follows:

$$
\begin{aligned}
\boldsymbol{\Sigma}_{1}^{0}(E) & =\text { open subsets of } E \\
\text { if } \alpha \geq 2 \boldsymbol{\Sigma}_{\alpha}^{0}(E) & =\text { countable unions of Boolean combinations of sets in } \bigcup_{\beta<\alpha} \boldsymbol{\Sigma}_{\beta}^{0}(E) \\
\boldsymbol{\Pi}_{\alpha}^{0}(E) & =\left\{E \backslash X \mid X \in \boldsymbol{\Sigma}_{\alpha}^{0}(E)\right\} \\
\boldsymbol{\Delta}_{\alpha}^{0}(E) & =\boldsymbol{\Sigma}_{\alpha}^{0}(E) \cap \boldsymbol{\Pi}_{\alpha}^{0}(E)
\end{aligned}
$$

3 The class $\mathbf{G}_{\delta}(E)$ (respectively $\mathbf{F}_{\sigma}(E)$ ) is the family of countable intersections of open 
sets (respectively unions of closed sets). In general, it is a proper subclass of $\boldsymbol{\Pi}_{2}^{0}(E)$ (respectively $\boldsymbol{\Sigma}_{2}^{0}(E)$ ).

The following result follows from elementary set theory.

\section{Proposition 2.2.}

$1 \quad \boldsymbol{\Sigma}_{\beta}^{0}(E) \cup \boldsymbol{\Pi}_{\beta}^{0}(E) \subseteq \boldsymbol{\Delta}_{\alpha}^{0}(E)$ for any $\alpha>\beta \geq 1$.

2 Each one of the Borel classes $\boldsymbol{\Sigma}_{\alpha}^{0}(E), \boldsymbol{\Pi}_{\alpha}^{0}(E), \boldsymbol{\Delta}_{\alpha}^{0}(E)$ is closed under finite unions and intersections and continuous inverse images. The $\boldsymbol{\Sigma}_{\alpha}^{0}(E)$ (respectively $\boldsymbol{\Pi}_{\alpha}^{0}(E)$ ) classes are closed under countable unions (respectively intersections).

As expected, the above definition is equivalent to the usual one for metric spaces. Also, in the general case, the distortion can be done solely for $\boldsymbol{\Sigma}_{2}^{0}(E)$.

\section{Proposition 2.3.}

$1 \boldsymbol{\Sigma}_{2}^{0}(E)$ coincides with the family of countable unions of differences of sets in $\boldsymbol{\Sigma}_{1}^{0}(E)$, i.e. sets of the form $\cup_{n \in \mathbb{N}} U_{n} \backslash V_{n}$ where the $U_{n}, V_{n}$ 's are open. Moreover, if $\mathcal{B}$ is a countable topological basis then one can take the $U_{n}$ 's in $\mathcal{B}$.

2 If $\alpha \geq 3$ then $\boldsymbol{\Sigma}_{\alpha}^{0}(E)$ is the family of countable unions of sets in $\bigcup_{\beta<\alpha} \Pi_{\beta}^{0}(E)$. If $E$ is metrizable then this also holds for $\alpha=2$.

Proof. 1. Observe that a Boolean combination of open sets is a finite union of differences of two open sets. For the last assertion, use that $U_{n}$ is a union of sets in $\mathcal{B}$.

2. It suffices to prove that the difference $X \backslash Y$ of two sets in $\boldsymbol{\Sigma}_{\beta}^{0}(E)$, with $\beta<\alpha$, is equal to a countable union of sets in $\Pi_{\gamma}^{0}(E)$ with $\gamma<\alpha$. In case $\beta+1<\alpha$ then, as the intersection of a $\boldsymbol{\Sigma}_{\beta}^{0}(E)$ and a $\boldsymbol{\Pi}_{\beta}^{0}(E)$ set, $X \backslash Y$ is $\boldsymbol{\Pi}_{\beta+1}^{0}(E)$ and we are done. In case $\alpha=\beta+1$, since $\alpha \geq 3$, we have $\beta \geq 2$ and $X$ is of the form $X=\bigcup_{i \in \mathbb{N}} U_{i} \backslash V_{i}$ where $U_{i}, V_{i}$ are in $\bigcup_{\gamma<\beta} \Sigma_{\gamma}^{0}(E)$. Thus, $X \backslash Y=\bigcup_{i \in \mathbb{N}}\left(U_{i} \backslash V_{i}\right) \backslash Y=\bigcup_{i \in \mathbb{N}} U_{i} \cap\left(E \backslash\left(V_{i} \cup Y\right)\right)$. Now, $U_{i} \in \Sigma_{\gamma}^{0}(E)$, with $\gamma<\beta$, hence $U_{i} \in \boldsymbol{\Pi}_{\gamma+1}^{0}(E)$ where $\gamma+1 \leq \beta$. Also, $V_{i} \cup Y \in \boldsymbol{\Sigma}_{\beta}^{0}(E)$ hence $E \backslash\left(V_{i} \cup Y\right) \in \boldsymbol{\Pi}_{\beta}^{0}(E)$. Therefore, $X \backslash Y$ is a countable union of sets in $\Pi_{\beta}^{0}(E)$. Finally, in a metric space, the topological closure $\bar{X}$ of any set $X$ is $\mathbf{G}_{\delta}$ since $\bar{X}=\bigcap_{n \in \mathbb{N}}\left\{z \mid \exists x \in X d(z, x)<2^{-n}\right\}$. Going to complements, any open set is $\mathbf{F}_{\sigma}$. Then, any difference of two open sets hence also any $\boldsymbol{\Sigma}_{2}^{0}(E)$ set is also $\mathbf{F}_{\sigma}$, i.e. a countable union of $\boldsymbol{\Pi}_{1}^{0}(E)$ sets.

2.1.2. The Hausdorff Difference Hierarchy. Recall the Hausdorff difference infinitary operation, cf. (Kuratowski 1966, Kechris 1995).

Definition 2.4. Let $\alpha$ be an ordinal.

1 The difference operation $D_{\alpha}$ maps an $\alpha$-sequence of subsets $\left(A_{\beta}\right)_{\beta<\alpha}$ of a space $E$ to the subset $D_{\alpha}\left(\left(A_{\beta}\right)_{\beta<\alpha}\right)=\bigcup_{\beta<\alpha, \beta \nmid \alpha} A_{\beta} \backslash \cup_{\gamma<\beta} A_{\gamma}$

2 We let $c o-D_{\alpha}\left(\left(A_{\beta}\right)_{\beta<\alpha}\right)=E \backslash D_{\alpha}\left(\left(A_{\beta}\right)_{\beta<\alpha}\right)$.

3 For a class of subsets $\mathcal{A}$, we let $\mathbf{D}_{\alpha}(\mathcal{A})$ (respectively co- $\mathbf{D}_{\alpha}(\mathcal{A})$ ) be the class of all subsets $D_{\alpha}\left(\left(A_{\beta}\right)_{\beta<\alpha}\right)$ (respectively co- $D_{\alpha}\left(\left(A_{\beta}\right)_{\beta<\alpha}\right)$ ), where $A_{\beta} \in \mathcal{A}$ for all $\beta<\alpha$.

Remark 2.5. In particular, $\mathbf{D}_{2}(\mathcal{A})$ (respectively co- $\mathbf{D}_{2}(\mathcal{A})$ ) is the family of sets $A_{1} \backslash A_{0}$ (respectively $A_{0} \cup\left(E \backslash A_{1}\right)$ ) with $A_{0}, A_{1} \in \mathcal{A}$. 
Fig. 1. In grey: $D_{1}\left(A_{0}\right), D_{2}\left(A_{0}, A_{1}\right), D_{3}\left(A_{0}, A_{1}, A_{2}\right), D_{4}\left(A_{0}, A_{1}, A_{2}, A_{3}\right)$ where $A_{0} \subset A_{1} \subset A_{2} \subset A_{3}$. In white (including the unbounded complement of the largest disk): co- $D_{1}\left(A_{0}\right)$, co- $D_{2}\left(A_{0}, A_{1}\right)$, co- $D_{3}\left(A_{0}, A_{1}, A_{2}\right)$, co- $D_{4}\left(A_{0}, A_{1}, A_{2}, A_{3}\right)$.

\section{Proposition 2.6.}

1 If $\varnothing \in \mathcal{A}$ then $\mathbf{D}_{\beta}(\mathcal{A}) \subseteq \mathbf{D}_{\alpha}(\mathcal{A})$ for all $\beta<\alpha$.

2 If $E \in \mathcal{A}$ then $c o$ - $\mathbf{D}_{\alpha}(\mathcal{A}) \subseteq \mathbf{D}_{\alpha+1}(\mathcal{A})$. In particular, if $\varnothing, E \in \mathcal{A}$ and $\beta<\alpha$ then $\mathbf{D}_{\beta}(\mathcal{A}) \cup c o-\mathbf{D}_{\beta}(\mathcal{A}) \subseteq \mathbf{D}_{\alpha}(\mathcal{A}) \cap c o-\mathbf{D}_{\alpha}(\mathcal{A})$.

3 If $\mathcal{A}$ is closed under countable unions then, for $\alpha$ countable, in the definition of $\mathbf{D}_{\alpha}(\mathcal{A})$, one can restrict to monotone increasing $\alpha$-sequences.

Proof. 1. If $\beta \sim \alpha$ then $D_{\beta}\left(\left(A_{\gamma}\right)_{\gamma<\beta}\right)=D_{\alpha}\left(\left(A_{\delta}^{\prime}\right)_{\delta<\alpha}\right)$ where $A_{\delta}^{\prime}=A_{\delta}$ for $\delta<\beta$ and $A_{\delta}^{\prime}=\varnothing$ for $\delta \geq \beta$. If $\beta \psi \alpha$ then $D_{\beta}\left(\left(A_{\gamma}\right)_{\gamma<\beta}\right)=D_{\alpha}\left(\left(A_{\delta}^{\prime}\right)_{\delta<\alpha}\right)$ where $A_{\delta+1}^{\prime}=A_{\delta}$ for $\delta<\beta$ and $A_{\delta}^{\prime}=\varnothing$ for $\delta=0$ or $\delta$ limit or $\delta \geq \beta$.

2. Observe that $D_{\alpha}\left(\left(A_{\beta}\right)_{\beta<\alpha}\right)=D_{\alpha}\left(\left(A_{\beta}^{\prime}\right)_{\beta<\alpha}\right)$ where $A_{\beta}^{\prime}=\cup_{\gamma \leq \beta} A_{\gamma}$.

3. Letting $A_{\alpha}=E$, we have $c o-D_{\alpha}\left(\left(A_{\beta}\right)_{\beta<\alpha}\right)=D_{\alpha+1}\left(\left(A_{\beta}\right)_{\beta \leq \alpha}\right)$.

Definition 2.7. For any $0<\beta<\omega_{1}$, the $\alpha$-th level of the difference hierarchy over $\boldsymbol{\Sigma}_{\beta}^{0}(E)$ is $\mathbf{D}_{\alpha}\left(\boldsymbol{\Sigma}_{\beta}^{0}(E)\right)$. The difference hierarchy over $\boldsymbol{\Sigma}_{1}^{0}(E)$ is simply called the difference hierarchy and denoted by $\mathbf{D}_{\alpha}(E)$.

\section{Remark 2.8.}

1 Using item 2 of Proposition 2.6. we can graphically represent sets in the first levels of the difference hierarchy as in Figure 1.

2 This graphical representation makes it clear that $\mathbf{D}_{\alpha}(E)$ is not closed under finite union nor finite intersection: for instance, if $A_{0} \subset A_{1} \subset A_{2}$ then

$$
\begin{array}{lccc} 
& D_{2}\left(\varnothing, A_{0}\right) \cup D_{2}\left(A_{1}, A_{2}\right) & = & D_{3}\left(A_{0}, A_{1}, A_{2}\right) \\
\text { hence } & c o-D_{2}\left(\varnothing, A_{0}\right) \cap c o-D_{2}\left(A_{1}, A_{2}\right) & = & c o-D_{3}\left(A_{0}, A_{1}, A_{2}\right) \\
\text { and } & D_{3}\left(\varnothing, A_{0}, E\right) \cap D_{3}\left(A_{1}, A_{2}, E\right) & = & D_{4}\left(A_{0}, A_{1}, A_{2}, E\right) .
\end{array}
$$

Proposition 2.9. $\cup_{\alpha<\omega_{1}} \mathbf{D}_{\alpha}\left(\Sigma_{\beta}^{0}(E)\right) \subseteq \boldsymbol{\Delta}_{\beta+1}^{0}(E)$.

Proof. If the $A_{\gamma}$ 's, $\gamma<\alpha$ are in $\boldsymbol{\Sigma}_{\beta}^{0}(E)$ then so are the $\bigcup_{\delta<\gamma} A_{\delta}$ 's. Thus, $D_{\alpha}\left(\left(A_{\gamma}\right)_{\gamma<\alpha}\right)$ is a countable union of differences of sets in $\boldsymbol{\Sigma}_{\beta}^{0}(E)$ hence is in $\boldsymbol{\Sigma}_{\beta+1}^{0}(E)$. By Proposition 2.6. we see that $E \backslash D_{\alpha}\left(\left(A_{\gamma}\right)_{\gamma<\alpha}\right)=D_{\alpha+1}\left(\left(A_{\gamma}\right)_{\gamma<\alpha}, E\right) \in \Pi_{\beta+1}^{0}(E)$. 
Proposition 2.10. Let $D, E$ be topological spaces and $f: D \rightarrow E$ be continuous. If $Y \subseteq E$ is in some Hausdorff class $\mathrm{D}_{\alpha}(E), \alpha<\omega_{1}$, then $f^{-1}(\mathcal{Y})$ is in $\mathrm{D}_{\alpha}(D)$.

2.1.3. The Borel and Hausdorff Hierarchies May Collapse. As it is well-known, the Borel and Hausdorff hierarchies are proper in uncountable Polish spaces: $\boldsymbol{\Sigma}_{\alpha}^{0}(E) \nsubseteq \boldsymbol{\Sigma}_{\beta}^{0}(E)$ and $\mathbf{D}_{\alpha}\left(\boldsymbol{\Sigma}_{\xi}^{0}(E)\right) \mp \mathbf{D}_{\beta}\left(\boldsymbol{\Sigma}_{\xi}^{0}(E)\right)$ when $\alpha<\beta$. The same for the effective hierarchies (with $\left.\alpha, \beta<\omega_{1}^{C K}\right)$ relative to some fixed enumeration of a countable basis of open sets. However, this is not true in general topological spaces. For instance, if the space is $T_{2}$ and countable then $\boldsymbol{\Sigma}_{2}^{0}(E)=\mathcal{P}(E)$. However, (de Brecht 2011) proves the non collapse of the Borel and Hausdorff difference hierarchies in uncountable quasi-Polish spaces, a class containing Polish spaces and $\omega$-continuous domains.

\subsection{Domains}

Domain theory refers to the field initiated by Dana Scott in the late 1960s to specify denotational semantics for functional programming languages. The theory formalizes the ideas of approximation and convergence via some partially ordered sets called domains.

Example 2.11. Some examples of Scott topologies on partially ordered sets.

1 Scott topology on $(\mathcal{P}(\mathbb{N}), \subseteq)$. For $A \in \mathcal{P}_{<\omega}(\mathbb{N})$, let $O_{A}=\{X \in \mathcal{P}(\mathbb{N}) \mid X \supseteq A\}$. The Scott topology on $\mathcal{P}(\mathbb{N})$ is has the $O_{A}$ 's, for $A \in \mathcal{P}_{<\omega}(\mathbb{N})$, as a topological basis. This is the topology of "positive information"; in contrast, the Cantor topology on $\mathbf{2}^{\omega}$ gives positive and negative information.

2 Scott topology on $\left(\mathcal{P}_{\infty}(\mathbb{N}), \subseteq\right)$ : consider the family $\mathcal{P}_{\infty}(\mathbb{N})$ of infinite subsets of $\mathbb{N}$ as a topological subspace of $\mathcal{P}(\mathbb{N})$.

3 Scott topology on the family $\left(X^{\leq \omega}, \leq\right.$ pref $)$ of finite or infinite $X$-sequences. We suppose $X$ is any set with at least two elementss. For any $s \in X^{<\omega}$, let $\mathcal{B}_{s}=\{u \in$ $X^{\leq \omega} \mid u$ extends $\left.s\right\}$. The Scott topology on the space $X^{\leq \omega}$ is that which admits the $\mathcal{B}_{s}$ 's, $s \in X^{<\omega}$, as a topological basis.

4 Scott topology on the right extended real line $(\overrightarrow{\mathbb{R}}, \leq)$. Let $\overrightarrow{\mathbb{R}}=\mathbb{R} \cup\{+\infty\}$. The open sets of the topology on $\overrightarrow{\mathbb{R}}$ are $\overrightarrow{\mathbb{R}}$ and the semi-intervals $] x,+\infty]$, for $x \in \mathbb{R}$.

5 Extended real line $(\widetilde{\mathbb{R}}, \leq)$ with duplicated rationals. Let $\widetilde{\mathbb{R}}=\mathbb{R} \cup(\mathbb{Q} \times\{+\}) \cup\{+\infty\}$ and $\leq$ be the following total order: $+\infty$ is a maximum element, and for all $x, y \in \mathbb{R}$ and $q \in \mathbb{Q}, \quad q<\widetilde{\mathbb{R}}(q,+) ; x<\widetilde{\mathbb{R}} y$ if and only if $x<y$; and $x<\widetilde{\mathbb{R}}(q,+)<\widetilde{\mathbb{R}} y$ if and only if $x<q<y$. The Scott topology on $\widetilde{\mathbb{R}}$ is that for which the $[(q,+),+\infty]$ 's, $q \in \mathbb{Q}$, are a topological basis.

The following properties are straightforward.

Proposition 2.12. The Scott topologies of spaces in Examples 2.11 are not $T_{2}$ but are $T_{0}$, i.e. they satisfy Kolmogorov's axiom: given two distinct points, one of them has a neighborhood which does not contain the other one (but this may not be symmetric).

\section{Remark 2.13.}

1 As noticed in (Selivanov 2005), the finite levels of the Scott Borel hierarchy on $\mathcal{P}(\mathbb{N})$ 
do not coincide with the corresponding ones on the Cantor space $\mathbf{2}^{\omega}: \boldsymbol{\Sigma}_{n}^{0}(\mathcal{P}(\mathbb{N})) \mp$ $\boldsymbol{\Sigma}_{n}^{0}\left(\mathbf{2}^{\omega}\right) \varsubsetneqq \boldsymbol{\Sigma}_{n+1}^{0}(\mathcal{P}(\mathbb{N}))$ for all $n \in \mathbb{N}$. The same is true with the effective Borel hierarchy (cf. $\S 5.3)$. For instance, $\mathcal{X}=\mathcal{P}(\mathbb{N}) \backslash\{\mathbb{N}\}$, defined by the formula $\exists x(x \notin X)$, is $\Sigma_{1}^{0}\left(\mathbf{2}^{\omega}\right)$ and $\Sigma_{2}^{0}(\mathcal{P}(\mathbb{N}))$ but neither Scott open nor Scott closed. However, the infinite levels of the Borel hierarchy on $\mathcal{P}(\mathbb{N})$ and $\mathbf{2}^{\omega}$ coincide.

2 The only subsets of $\mathcal{P}(\mathbb{N})$ that are both open and $F_{\sigma}$ are $\varnothing$ and $\mathcal{P}(\mathbb{N})$. Indeed, suppose $\mathcal{O}$ is open and $\mathcal{X}$ is $F_{\sigma}$ and $\mathcal{O}, \mathcal{X}$ are different from $\varnothing, \mathcal{P}(\mathbb{N})$. Then there exist non empty finite subsets $X, Y$ of $\mathbb{N}$ such that $O_{X} \subseteq \mathcal{O}$ and $\mathcal{P}(\mathbb{N}) \backslash O_{Y} \subseteq \mathcal{X}$. Observe that the set $X \cup Y$ is in $\mathcal{O} \backslash \mathcal{X}$, showing $\mathcal{O} \neq \mathcal{X}$.

2.2.1. The Scott Topology on dcpo's. We briefly recall the main definitions and notions and refer the reader to classical papers and books, for instance (Abramsky \& Jung 1994. Edalat 1997; Gierz \& al. 2003).

\section{Definition 2.14.}

1 A directed complete partial order (dcpo) is a partially ordered set $(D, \sqsubseteq)$ such that every non empty directed subset $S$ has a least upper bound (denoted by $\sqcup S$ ). A dcpo is pointed if it has a least element $\perp$.

2 The Scott topology on a dcpo is the topology that admits as closed sets all sets $X$ satisfying conditions

$-X$ is a downset: $x \in X \wedge y \sqsubseteq x \Rightarrow y \in X$.

$-X$ is closed under suprema of directed subsets of $D$.

Then, $O \subseteq D$ is open in the Scott topology if it satisfies the following conditions.

- $O$ is an upset: $x \in O \wedge x \sqsubseteq y \Rightarrow y \in O$.

- Every directed set with supremum in $O$ has an element in $O$.

Example 2.15. For every $x \in D$, the set $U_{x}=\{z \mid z \not x\}$ is Scott open.

\section{Proposition 2.16.}

1 The Scott topology on a dcpo is $T_{0}$, i.e. if $x \neq y$ then there exists an open set which contains only one of the two points $x, y$. It is $T_{1}$ (respectively $T_{2}$ ) if and only if the order on $D$ is trivial.

2 Let $x, y \in D$. The order on $D$ can be recovered from the topology as the specialization order: $x \sqsubseteq y$ if and only if every Scott open set containing $x$ also contains $y$ if and only $x \in \overline{\{y\}}$ (where $\overline{\{y\}}$ is the topological closure of $\{y\}$ ).

3 A function $f: D \rightarrow E$ between two dcpo's is continuous with respect to the Scott topologies if and only if it is monotone increasing and preserves suprema of directed subsets: if $S \subseteq D$ is directed then $f(\sqcup S)=\sqcup f(S)$.

\subsubsection{The Scott Topology on Domains.}

\section{Definition 2.17.}

1 Let $(D, \sqsubseteq$ ) be a dcpo. The approximation (or way-below) relation on $D$ is defined as 
follows: let $x, y \in D, x \ll y$ if, for all directed subset $S, y \sqsubseteq \sqcup S$ implies $x \sqsubseteq s$ for some $s \in S$. We say $x$ approximates, or is way-below, $y$.

2 An element $x \in D$ is compact (or finite) if $x \ll x$. The set of compact elements is denoted by $K(D)$.

$3 \uparrow x=\{y \mid x \ll y\}$ and $\downarrow x=\{y \mid y \ll x\}$

Proposition 2.18. Let $\left(D\right.$, ㄷ) be a dcpo and $x, x^{\prime}, y, y^{\prime} \in D$.

$1 \quad x \ll y \Rightarrow x \sqsubseteq y$, and $\left(x^{\prime} \sqsubseteq x \ll y \sqsubseteq y^{\prime}\right) \Rightarrow x^{\prime} \ll y^{\prime}$.

If $x$ is compact then $\forall u, v((u \sqsubseteq x \sqsubseteq v) \Leftrightarrow(u \ll x \ll v))$.

2 An element $x$ is compact if and only if $\uparrow x=\{y \mid x \sqsubseteq y\}$ is Scott open.

Proposition 2.19. Let $(D, \sqsubseteq)$ be a dcpo. The following conditions are equivalent.

(i) For every $x \in D$, the set $\downarrow x=\{z \in D \mid z \ll x\}$ is directed and $x=\sqcup \downarrow x$.

(ii) There exists $B \subseteq D$ such that, for every $x \in D, B \cap \downarrow x$ is directed and $x=\sqcup(B \cap \downarrow x)$.

$D$ is a continuous domain if these conditions hold. Any set $B$ satisfying condition (ii) is called a basis. $D$ is an $\omega$-continuous domain if (ii) holds for some countable set $B . D$ is an algebraic (respectively $\omega$-algebraic) domain if $K(D)$ is a basis (and is countable).

Example 2.20. All spaces in Example 2.11 are dcpo's with the Scott topology. The spaces $\mathcal{P}(\mathbb{N}), X^{\leq \omega}, \widetilde{\mathbb{R}}$ are $\omega$-algebraic domains. Their sets of compact elements are respectively $\mathcal{P}_{<\omega}(\mathbb{N}), X^{<\omega}$ and $\mathbb{Q} \times\{+\}$. The way-below relation on any of these three spaces

is the restriction of the partial order $\leq$ to $K(D) \times D$. The space $\overrightarrow{\mathbb{R}}$ is an $\omega$-continuous domain but is not algebraic: there is no compact element and its way-below relation is the strict order $<$. The space $\mathcal{P}_{\infty}(\mathbb{N})$ is not continuous: its way-below relation is empty.

Let us recall classical results in continuous domains.

Proposition 2.21. Let $(D, \sqsubseteq)$ be a continuous domain with basis $B$.

1 (Interpolation property). If $M \subseteq D$ is finite and $a \ll x$ for each $a \in M$ then there exists $x^{\prime} \in B$ such that $M \ll x^{\prime} \ll x$.

$2 x \ll y$ if and only if $y$ is interior to the upper cone $\uparrow x=\{z \mid x \sqsubseteq z\}$.

3 A set $O$ is open if and only if $O=\bigcup_{x \in O} \uparrow x$ if and only if $O=\bigcup_{x \in O \cap B} \uparrow x$. In particular, the family of sets $\uparrow z$, where $z$ varies in $B$, is a basis of the Scott topology on $D$.

\subsection{Quasi-Polish Spaces}

Quasi-Polish spaces, developed by de Brecht (2011), are a unifying framework of Polish spaces and $\omega$-continuous domains. We recall here the definition and main results.

\section{Definition 2.22.}

1 Giving up the symmetry axiom of metrics, a quasi-metric on a space $E$ is defined as a function $d: E^{2} \rightarrow[0,+\infty[$ such that, for all $x, y, z \in E$,

$$
x=y \Leftrightarrow d(x, y)=d(y, x)=0 \quad, \quad d(x, z) \leq d(x, y)+d(y, z) .
$$

The topology associated to $d$ is the one generated by the open balls. 
2 A sequence $\left(x_{n}\right)_{n \in \mathbb{N}}$ is Cauchy if $\lim _{n \rightarrow+\infty} \sup _{p \geq n} d\left(x_{n}, x_{p}\right)=0$.

3 A quasi-metric space $(E, d)$ is complete if every Cauchy sequence is convergent relative to the metric $\widehat{d}$ such that $\widehat{d}(x, y)=\max \{d(x, y), d(y, x)\}$.

4 Topological spaces associated to complete quasi-metrics with a countable topological basis are called quasi-Polish.

Example 2.23. The Scott topology on $\mathcal{P}(\mathbb{N})$ is quasi-Polish for the quasi-metric such that $d(X, Y)=\sum_{n \in X \backslash Y} 2^{-n}$.

Theorem 2.24. (Künzi 1983) A quasi-metric space $(E, d)$ has a countable topological basis if and only if the metric space $(E, \widehat{d})$ is separable (i.e. has a countable dense subset).

The following theorem sums up some of the main results in (de Brecht 2011).

Theorem 2.25. de Brecht 2011).

1 A space is quasi-Polish if and only if it is homeomorphic to some $\boldsymbol{\Pi}_{2}^{0}$ subspace of $\mathcal{P}(\mathbb{N})$ endowed with the Scott topology.

2 Polish spaces and $\omega$-continuous domains are quasi-Polish.

3 Every quasi-Polish space $E$ satisfies the following properties:

- (Baire property) The intersection of a sequence of dense open sets is a dense set.

- (Hausdorff-Kuratowski property) $\cup_{\alpha<\omega_{1}} \mathbf{D}_{\alpha}\left(\Sigma_{\beta}^{0}(E)\right)=\Delta_{\beta+1}^{0}(E)$ for all $0<\beta<\omega_{1}$.

Remark 2.26. Two examples of subspaces of $\mathcal{P}(\mathbb{N})$ illustrate the above theorem.

1 The subspace $C=\{X \in \mathcal{P}(\mathbb{N}) \mid \forall i \in \mathbb{N}(2 i \in X \Leftrightarrow 2 i+1 \notin X)\}=\bigcap_{i \in \mathbb{N}}\left(O_{2 i} \Delta O_{2 i+1}\right)$ is $\boldsymbol{\Pi}_{2}^{0}$ in $\mathcal{P}(\mathbb{N})$ and is homeomorphic to the Cantor space $\mathbf{2}^{\omega}$ (and is therefore Polish).

2 The subspace $\mathcal{P}_{\infty}(\mathbb{N})=\bigcap_{i \in \mathbb{N}} \cup_{j \geq i} O_{\{j\}}$ is $\Pi_{2}^{0}$ in $\mathcal{P}(\mathbb{N})$ hence is quasi-Polish. It has a countable basis but is not Polish since it is $T_{0}$ and not $T_{2}$. Although $\left(\mathcal{P}_{\infty}(\mathbb{N}), \subseteq\right)$ is a $\mathrm{dcpo}$, it is not a continuous domain since its way-below relation is empty.

\subsection{Topological Games}

The Choquet topological games have been used to characterize Polish spaces (Choquet 1969) and quasi-Polish spaces (de Brecht 2011). We shall use them to characterize approximation spaces (cf. Definition 3.1 infra). Choquet games are a variant of Banach-Mazur games. The subtlety of this variant is best understood by confronting definitions and properties of both classes of games. Also, the interest of de Brecht's Theorem 2.36 and our Corollary 3.12 about convergent Markov/stationary strategies in the Choquet game is highlighted by the counterpart (and more powerful) results by Galvin \& Telgársky for Banach-Mazur games (cf. Corollary 2.34, Remark 2.35).

2.4.1. Banach-Mazur and Choquet Games. Let us recall the classical definitions.

Definition 2.27. (Choquet 1969 Galvin \& Telgárky 1986) Let $X$ be a topological space.

1 In the Banach-Mazur game $B M(X)$ two players, Empty and Nonempty, alternate turns for $\omega$ rounds. On round 0 (respectively $i+1$ ), Empty moves first, choosing a non empty open subset $U_{0} \subseteq X$ (respectively $\left.U_{i+1} \subseteq V_{i}\right)$. Then, Nonempty responds 
with a non-empty open set $V_{0} \subseteq U_{0}$ (respectively $V_{i+1} \subseteq U_{i+1}$ ). After all the rounds have been played, Nonempty wins if $\bigcap_{i \in \mathbb{N}} V_{i} \neq \varnothing$. Otherwise, Empty wins.

2 The Choquet game $C h(X)$ is the variant of the Banach-Mazur game $B M(X)$ where at round $i$ Empty picks a pair $\left(x_{i}, U_{i}\right)$ such that $U_{i}$ is open and $x_{i} \in U_{i}$, and Nonempty picks an open set $V_{i}$ such that $x_{i} \in V_{i} \subseteq U_{i}$.

3 A winning strategy for a player (in any of the above games) is a function that takes a partial play of the game ending with a move by its opponent and returns a move to play, such that the player wins any play of the game that follows the strategy.

4 A winning strategy for Nonempty is convergent if, when he follows it, the $V_{i}$ 's are a basis of neighborhoods of some $x \in \bigcap_{i \in \mathbb{N}} V_{i}$.

Note 2.28. The denominations "Banach-Mazur" and "Choquet" are the most commonly used (and are historically accurate). However, in (Kechris 1995) these games are respectively called "Choquet" and "strong Choquet".

Remark 2.29. Every winning strategy for Nonempty in the Choquet game $C h(X)$ yields one in the Banach-Mazur game $B M(X)$.

The following theorem sums up some known results around these topological games.

Theorem 2.30. Let $X$ be a topological space.

1 (Oxtoby 1957, cf. Kechris 1995, Theorem 8.11) $X$ has the Baire property (i.e. the intersection of countably many dense open sets is dense) if and only if player Empty has no winning strategy in the Banach-Mazur game $B M(X)$.

2 (Choquet 1969, cf. Kechris 1995, Theorem 8.18) $X$ is Polish if and only if $X$ has a countable basis, is $T_{1}$ and regular and player Nonempty has a winning strategy in the Choquet game $C h(X)$.

3 (de Brecht 2011, Theorem 51) $X$ is quasi-Polish if and only if $X$ has a countable basis, and player Nonempty has a convergent winning strategy in the Choquet game $C h(X)$.

2.4.2. Markov and Stationary Strategies. The pioneer work of Schmidt (1966) and Choquet (1969) considered strategies of a very simple form. Then Galvin \& Telgárky (1986) obtained deep results for other strategies.

Definition 2.31. A winning strategy is stationary (respectively Markov) if it depends only on the last move of the opponent (respectively and on the ordinal rank of the round).

Theorem 2.32. (Galvin \& Telgárky 1986, Theorems 5 and 7$)$ Let $(S, \leq)$ be a non empty partially ordered set. Let $R$ be a family of monotone (non strictly) decreasing sequences in $S$. In the game $G(S, \leq, R)$, player I starts and plays elements $a_{0}, a_{1}, \ldots$ of $S$, player II plays elements $b_{0}, b_{1}, \ldots$ of $S$ in such a way that $a_{i+1} \leq b_{i}$ and $b_{i} \leq a_{i}$ for all $i$. Player II wins if and only if $\left(a_{0}, a_{1}, \ldots\right) \in R$. Suppose $R$ is such that for all monotone (non strictly) decreasing sequences $\left(x_{i}\right)_{i \in \mathbb{N}},\left(y_{i}\right)_{i \in \mathbb{N}}$ in $S$,

$$
\left(\forall i \exists j y_{j} \leq x_{i}\right) \wedge\left(\forall j \exists k x_{k} \leq y_{j}\right) \wedge\left(x_{i}\right)_{i \in \mathbb{N}} \in R \Rightarrow\left(y_{i}\right)_{i \in \mathbb{N}} \in R .
$$


1 If player II has a winning strategy in $G(S, \leq, R)$ then he has one that depends only on the last move of I and the last move of II.

2 If player II has a Markov winning strategy in $G(S, \leq, R)$ then he has a stationary one.

Corollary 2.33. (Galvin \& Telgárky 1986, Corollaries 9 and 14)

1 If player Nonempty has a winning (respectively and convergent) strategy in the Banach-Mazur game $B M(X)$ then he has one which depends only on the last move of Empty and the last move of Nonempty (respectively and is convergent).

2 If player Nonempty has a Markov (respectively and convergent) winning strategy in $B M(X)$ then he has a stationary (respectively and convergent) one.

3 (Debs 1984, 1985) There exists a $T_{2}$ (even completely regular) space $X$ such that player Nonempty has no stationary winning strategy in $B M(X)$ but has a winning strategy which depends only on the last two moves of Empty.

Remark 2.34. Theorem 2.32 does not apply to the Choquet game because the players do not play in the same partially ordered set. For simple winning strategies for Nonempty in the Choquet game $C h(X)$ in particular spaces $X$, see $\S 5$ of (Bennett \& Lutzer 2009).

The proof of item 3 of Theorem 2.30 given by de Brecht (Theorem 51 in de Brecht 2011) yields more:

Theorem 2.35. (de Brecht 2011) If $X$ is quasi-Polish then player Nonempty has a Markov convergent winning strategy in the Choquet game $C h(X)$.

We shall improve this last result (replacing Markov by stationary), cf. Corollary 3.13

\section{Approximation Spaces: the Spaces of Choquet Games}

\subsection{Approximation Spaces}

We introduce another class of topological spaces: approximation spaces. They include all continuous domains, all Polish spaces and, in fact, all quasi-Polish spaces. The definition is based on an approximation relation which formalizes a containment relation between basic open sets. This containment relation ensures that inclusion-decreasing chains have a non empty intersection; however, this intersection may not be reduced to a singleton set. An example of an approximation relation is obtained by lifting to basic open sets the way-below relation in a dcpo. We borrowed the notation $\ll$ from this particular example.

Theorem 3.12 proves that a subclass of second-countable approximation spaces coincides with the class of quasi-Polish spaces. This gives an "à la domain" characterization of quasi-Polish spaces. Whether the notion of approximation space captures a substantial part of the rich theory developed by de Brecht for quasi-Polish spaces is a question still

to be investigated. In the next sections, we just show two pleasant properties: a $\Pi_{2}^{0}$ Baire property and Hausdorff's characterization of $\boldsymbol{\Delta}_{2}^{0}$.

Definition 3.1. Let $E$ be a topological space.

- An approximation relation for $E$ is a binary relation $\ll$ on some topological basis $\mathcal{B}$ such that, for all $U, V, T \in \mathcal{B}$, 
(1) If $U \ll V$ then $V \subseteq U$,

(2) If $U \subseteq T$ and $U \ll V$ then $T \ll V$ (in particular, $\ll$ is transitive),

(3) for all $x \in U$, there exists $W \in \mathcal{B}$ such that $x \in W$ and $U \ll W$,

(4) For every sequence $\left(U_{i}\right)_{i \in \mathbb{N}}$ of sets in $\mathcal{B}$ such that $U_{i} \ll U_{i+1}$ for all $i$, the intersection set $\bigcap_{i \in \mathbb{N}} U_{i}$ is non empty.

- Convergent approximation relations are obtained by strengthening condition (4) to

$\left(4^{+}\right)$Every sequence $\left(U_{i}\right)_{i \in \mathbb{N}}$ of sets in $\mathcal{B}$ such that $U_{i} \ll U_{i+1}$ for all $i$, is a neighborhood basis of some $x \in \bigcap_{i \in \mathbb{N}} U_{i}$ (i.e. each open set containing $x$ also contains some $U_{i}$ ).

- An approximation relation is hereditary if, for every closed subset $C$ of $E$,

$$
\ll_{C}=\{(C \cap U, C \cap V) \mid U \ll V \text { and } C \cap U, C \cap V \neq \varnothing\}
$$

is an approximation relation for the subspace $C$.

- A space $E$ is an approximation space (respectively convergent approximation space, respectively hereditary approximation space) if it admits an approximation (respectively convergent approximation, respectively hereditary approximation) relation.

Example 3.2. Every subspace $D$ of the $\operatorname{Scott}$ domain $\mathcal{P}(\mathbb{N})$ which is an upset (i.e. if $X \subseteq Y$ and $X \in D$ then $Y \in D)$ is a trivial approximation space: containment is an approximation relation on the basis $\left\{D \cap O_{A} \mid \alpha \in \mathcal{P}_{<\omega}(\mathbb{N})\right\}$ where $O_{A}=\{X \mid A \subseteq X\}$ since $\mathbb{N}$ belongs to all $D \cap O_{A}$ 's. In particular, the dcpo $\left(\mathcal{P}_{\infty}(\mathbb{N}), \subseteq\right)$ is an approximation space which is neither Polish nor a continuous domain. $\operatorname{In} \mathcal{P}(\mathbb{N})$, inclusion is a convergent approximation relation since $\bigcup_{i \in \mathbb{N}} A_{i} \in \bigcap_{i \in \mathbb{N}} O_{A_{i}}$ and the $O_{A_{i}}$ 's converge to $\bigcup_{i \in \mathbb{N}} A_{i}$.

Approximation relations exist on all topological basis or on none.

Lemma 3.3. Let $\mathcal{B}$ and $\mathcal{C}$ be topological basis of a space $E$. If there exists an (respectively convergent; respectively hereditary) approximation relation on $\mathcal{B}$ then there exists one on $\mathcal{C}$.

Proof. Let $\ll$ be an approximation relation on $\mathcal{B}$. Consider the relation $\ll$ on $\mathcal{C}$ such that, for any $C, D \in \mathcal{C}$,

$$
\text { (*) } \quad C \ll D \Longleftrightarrow \exists U, V \in \mathcal{B}(C \supseteq U \ll V \supseteq D) .
$$

Let us check that « satisfies conditions (1) to (4) of Definition 3.1. Conditions (1) and (2) are straightforward. We now look at Condition (3). Suppose $x \in C \in \mathcal{C}$. Let $U \in \mathcal{B}$ be such that $x \in U \subseteq C$. Applying condition (3) for $\ll$, there exists $V \in \mathcal{B}$ such that $x \in V$ and $U \ll V$. Let $D \in \mathcal{C}$ be such that $x \in D$ and $D \subseteq V$. Then $C \ll D$ so that condition (3) holds for «. Finally, we check Condition (4). Suppose $C_{i} \ll C_{i+1}$ for all $i$. Let $U_{i}, V_{i} \in \mathcal{B}$ be such that $C_{i} \supseteq U_{i} \ll V_{i} \supseteq C_{i+1}$. In particular, $V_{i} \supseteq U_{i+1} \ll V_{i+1}$ hence $V_{i} \ll V_{i+1}$ by condition (2) for $\ll$. Applying condition (4) for $\ll$, we see that $\bigcap_{i \in \mathbb{N}} C_{i}=\bigcap_{i \in \mathbb{N}} V_{i} \neq \varnothing$ so that condition (4) holds for $\ll$.

In case $\ll$ is convergent then the $V_{i}$ 's are a neighborhood basis of some $x \in \bigcap_{i \in \mathbb{N}} V_{i}$. Hence so are the $C_{i+1}$ 's and « is convergent. Suppose $\ll$ is hereditary. To see that $\ll$ is also hereditary, observe that, for any closed subset $F$ of $E$, the relation $\ll_{F}$ is obtained from $\ll_{F}$ via condition $(*)$. 
Proposition 3.4. Any convergent approximation relation is hereditary convergent.

Proof. Suppose $\left(U_{i}\right)_{i \in \mathbb{N}}$ is «-increasing and $C$ meets each $U_{i}$. Since « is a convergent approximation relation, the $U_{i}$ 's are a neighborhood basis of some $x \in \bigcap_{i \in \mathbb{N}} U_{i}$. Since the $U_{i}$ 's meet $C, x$ is adherent to $C$, hence it is in $C$. Also, the $U_{i} \cap C$ 's are a neighborhood basis of $x$ in the subspace $C$.

\subsection{Approximation Spaces versus quasi-Polish Spaces}

Proposition 3.5. Polish spaces and continuous domains are convergent approximation spaces.

Proof. Case of Polish spaces. Let $\mathcal{B}$ be the basis consisting of open balls centered in some fixed countable dense set and having rational radius. For $U, V \in \mathcal{B}$, let $U \ll V$ if and only if $\bar{V} \subseteq U$ and $\operatorname{diam}(V) \leq \operatorname{diam}(U) / 2\}$, where $\bar{V}$ is the topological closure of $V$ and diam is the diameter. All wanted conditions on $\ll$ are straightforward.

Case of continuous domains. Let $B$ be a basis in the sense of continuous domains. The family $\mathcal{B}=\{\uparrow b \mid b \in B$ and $\uparrow b \neq \varnothing\}$ is a topological basis. Define the relation $\ll$ on $\mathcal{B}$ as $U \ll V$ if and only if $V=\uparrow c$ for some $c \in U$. To check condition (1), observe that if $U=\uparrow b$ then $c \in U$ yields $b \ll c$, so that $U=\uparrow b \supseteq \uparrow c=V$. As for condition (2), if $W \supseteq U \ll V$ and $V=\uparrow c$ with $c \in U$ then $c \in W$ hence $W \ll V$. As for condition (3), let $x \in U=\uparrow b$, i.e. $b \ll x$. Using the interpolation property, let $c$ be such that $b \ll c \ll x$ and set $W=\uparrow c$. Then $x \in W$ and $c \in \uparrow b=U$, so that $U \ll W$. Finally, for condition $\left(4^{+}\right)$, suppose $U_{i} \ll U_{i+1}$ for all $i \in \mathbb{N}$ and choose $b_{i+1} \in U_{i}$ such that $U_{i+1}=\uparrow b_{i+1}$. Since $b_{i+2} \in U_{i+1}=\uparrow b_{i+1}$, we have $b_{i+1} \ll b_{i+2}$. Let $x$ be the supremum of the $b_{j}$ 's for $j \geq 1$. Then $x \in \bigcap_{j \geq 1} \uparrow b_{j}=\bigcap_{i \in \mathbb{N}} U_{i}$ which is therefore a non empty set. Also, the $\uparrow b_{j}=U_{j}$ 's, $j \geq 1$, are a basis of neighborhoods of $x$.

Using de Brecht's Theorem 2.25 for second-countable spaces, the above Proposition is partly subsumed by the next theorem.

Theorem 3.6. Quasi-Polish spaces are convergent approximation spaces.

Proof. By de Brecht's result stated in item 1 of Theorem 2.25] it suffices to show that any $\Pi_{2}^{0}$ subspace $\mathcal{A}$ of $\mathcal{P}(\mathbb{N})$ (with the Scott topology) is a convergent approximation space. In this proof we use Greek letters to denote finite sets. For $\alpha \in \mathcal{P}_{<\omega}(\mathbb{N})$, let $O_{\alpha}=$ $\{X \mid \alpha \subseteq X\}$. As a basis $\mathcal{B}$ of the subspace $\mathcal{A}$, we consider those $B_{\alpha}=O_{\alpha} \cap \mathcal{A}, \alpha \in \mathcal{P}_{<\omega}(\mathbb{N})$, which are non empty. Observe that if $U \in \mathcal{B}$, there may be infinitely many $\alpha$ 's such that $U=B_{\alpha}$. The family $\mathcal{A}$ is of the form $\mathcal{A}=\bigcap_{n \in \mathbb{N}}\left(U_{n} \cup F_{n}\right)$ where $U_{n}=\bigcup_{\alpha \in I_{n}} O_{\alpha}$ is open in $\mathcal{P}(\mathbb{N})$ and $F_{n}$ is closed in $\mathcal{P}(\mathbb{N})$. Since $F_{n}$ is a countable intersection of closed sets of the form $\mathcal{P}(\mathbb{N}) \backslash O_{\alpha}$, by merging this intersection with the global one, we can reduce to the case where $F_{n}=\mathcal{P}(\mathbb{N}) \backslash O_{\alpha_{n}}$ with $\alpha_{n} \in \mathcal{P}_{<\omega}(\mathbb{N})$. Then,

$$
X \in \mathcal{A} \Leftrightarrow \forall n\left(\alpha_{n} \nsubseteq X \vee \exists \gamma \in I_{n} \gamma \subseteq X\right) \Leftrightarrow \forall n\left(\alpha_{n} \subseteq X \Rightarrow \exists \gamma \in I_{n} \gamma \subseteq X\right) .
$$

Let us call clause $n$ the clause $\alpha_{n} \subseteq X \Rightarrow \exists \gamma \in I_{n} \gamma \subseteq X$. Thus, a set $X \subseteq \mathbb{N}$ is in $\mathcal{A}$ if and only if it satisfies clause $n$ for all $n$. We introduce two notions: 
- Clause $n$ is a $U$-clause if $U \subseteq O_{\alpha_{n}}$ (i.e. the premiss $\alpha_{n} \subseteq X$ of clause $n$ is satisfied by all $X \in U)$,

- Clause $n$ is $U$-solved if $U \subseteq O_{\gamma}$ for some $\gamma \in I_{n}$. (i.e. the conclusion $\exists \gamma \in I_{n} \gamma \subseteq X$ of clause $n$ is satisfied by all $X \in U$ with the same witness $\gamma$ ).

Observe that if $W \supseteq U$ then any $W$-clause is a $U$-clause and any $W$-solved $W$-clause is a $U$-solved $U$-clause. We denote by $n_{U}$ the least $n$ such that clause $n$ is a $U$-unsolved $U$-clause or $+\infty$ if there is no such clause. We now define the relation $\ll$ on $\mathcal{B}$ : for $U, V \in \mathcal{B}$,

$$
U \ll V \Longleftrightarrow V \subseteq U \text { and } \begin{cases}(i) & \text { either } n_{U}=+\infty \\ (i i) & \text { or } n_{U}<+\infty \text { and clause } n_{U} \text { is } V \text {-solved } \\ (\text { iii }) & \text { or } n_{U}<+\infty \text { and, for some } m<n_{U} \text {, clause } m \\ & \text { is not a } U \text {-clause and is a } V \text {-solved } V \text {-clause. }\end{cases}
$$

We check conditions (1), (2), (3) and $\left(4^{+}\right)$of Definition 3.1. Condition (1) is trivial. Condition (2). Suppose $W \supseteq U \ll V$. If $n_{W}=+\infty$ then $W \ll V$ holds by condition (i). So we shall suppose $n_{W}<+\infty$. Suppose $n_{W}<n_{U}$. Then clause $n_{W}$, being a $W$-clause hence a $U$-clause, is $U$-solved (by definition of $n_{U}$ ), hence it is also $V$-solved (since $U \supseteq V$ ), so that $W \ll V$ holds by condition (ii). We now assume $n_{U} \leq n_{W}<+\infty$. Since $n_{U}$ is finite, $U \ll V$ cannot hold by condition (i). If $U \ll V$ holds by condition (iii) then the witnessing clause $m$ is not a $U$-clause hence is not a $W$-clause, so that $W \ll V$ holds by condition (iii). Suppose now that $U \ll V$ holds by condition (ii). If $n_{W}=n_{U}$ then $W \ll V$ also holds by condition (ii). Suppose $n_{W}>n_{U}$. If clause $n_{U}$ were a $W$-clause then it would be $W$-solved (by definition of $n_{W}$ and inequality $n_{U}<n_{W}$ ) hence it would be a $U$-solved $U$-clause, contradicting the definition of $n_{U}$. Thus, clause $n_{U}$ is not a $W$-clause and $W \ll V$ holds by condition (iii).

Condition (3). Let $X$ be in $U \in \mathcal{B}$. If $n_{U}=+\infty$ then it suffices to set $V=U$. Suppose now that $n_{U}<+\infty$. Since clause $n_{U}$ is a $U$-clause and $X \in U$, we have $\alpha_{n_{U}} \subseteq X$ so that $X$ satisfies the premiss of clause $n_{U}$. Then, $X$ being in $\mathcal{A}$, satisfies all clauses, in particular clause $n_{U}$. So, there exists some $\gamma \in I_{n_{U}}$ such that $\gamma \subseteq X$. Now, $U \in \mathcal{B}$ hence $U=\mathcal{A} \cap O_{\beta}$ for some $\beta \in \mathcal{P}_{<\omega}(\mathbb{N})$. Set $V=U \cap O_{\gamma}$. Then $V=\mathcal{A} \cap O_{\beta} \cap O_{\gamma}=\mathcal{A} \cap O_{\beta \cup \gamma} \in \mathcal{B}$. Also, $X \in V$ since $X \in U$ and $\gamma \subseteq X$. Finally, $V$ solves clause $n_{U}$, hence $U \ll V$ by condition (ii) of $\ll$.

Condition (4). Let $\left(U_{i}\right)_{i \in \mathbb{N}}$ be a sequence of families in $\mathcal{B}$ such that $U_{i} \ll U_{i+1}$ for all $i$. Let $\beta_{i}$ be any set in $\mathcal{P}_{<\omega}(\mathbb{N})$ such that $U_{i}=\mathcal{A} \cap O_{\beta_{i}}$. Set $\delta_{i}=\bigcup_{j \leq i} \beta_{j}$ (so that the $\delta_{i}$ 's are increasing with $i$ ). Since $U_{j} \supseteq U_{i}$ for $j<i$, we have $U_{i}=\mathcal{A} \cap \bigcap_{j \leq i} O_{\beta_{i}}=\mathcal{A} \cap O_{\delta_{i}}$. To finish the proof, we show that the set $X=\bigcup_{i \in \mathbb{N}} \delta_{i}$ is in the family $\bigcap_{i \in \mathbb{N}} U_{i}=\mathcal{A} \cap \bigcap_{i \in \mathbb{N}} O_{\delta_{i}}$ (which is therefore non empty). Clearly, $X \in \bigcap_{i \in \mathbb{N}} O_{\delta_{i}}$. To show that $X \in \mathcal{A}$, i.e. $X$ satisfies clause $n$ for all $n$, we argue by contradiction. Suppose clause $n$ is the first clause not satisfied by $X$. Then, $X$ satisfies clause $m$ for all $m<n$. This means that if $\alpha_{m} \subseteq X$ then there is $\gamma_{X, m} \in I_{m}$ such that $\gamma_{X, m} \subseteq X$. Also, $X$ satisfies the premiss of clause $n$ (but not its conclusion), i.e. $\alpha_{n} \subseteq X$. Since $\alpha_{n}$ and those $\alpha_{m}$ 's, $\gamma_{X, m}$ 's included in $X$ (for $m<n$ ) are finite, they are all included in $\delta_{i}$ for some $i$. Thus,

- for each $m<n$, clause $m$ is a $U_{i}$-clause if and only if it is a $U_{i+1}$-clause (if and only if $\left.\alpha_{m} \subseteq X\right)$, 


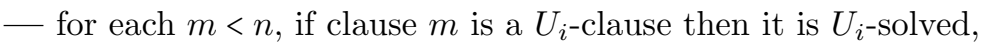

— clause $n$ is a $U_{i}$-clause and is not $U_{j}$-solved for any $j \geq i$.

Then, clause $n$ is the first $U_{i}$-unsolved $U_{i}$-clause and no clause $m, m<n$, can be a $U_{i+1^{-}}$ solved $U_{i+1}$-clause without being a $U_{i}$-clause. As a consequence, the assumed property $U_{i} \ll U_{i+1}$ necessarily comes from condition (ii) in the definition of $\ll$, i.e. clause $n$ is $U_{i+1}$-solved. Since $X \in U_{i+1}$, clause $n$ is satisfied by $X$. This is a contradiction.

Condition $\left(4^{+}\right)$. To show that the $U_{i}$ 's are a basis of neighborhoods of $X$ in $\mathcal{A}$, it suffices to prove that, for all $\beta \in \mathcal{P}_{<\omega}(\mathbb{N})$ such that $X \in O_{\beta}$, there exists $i$ such that $\mathcal{A} \cap O_{\beta} \subseteq \mathcal{A} \cap O_{\delta_{i}}$. Since $X=\bigcup_{i \in \mathbb{N}} \delta_{i} \in O_{\beta}$ we have $\beta \subseteq \delta_{i}$ for some $i$ hence $O_{\delta_{i}} \subseteq O_{\beta}$ and the wanted inclusion $\mathcal{A} \cap O_{\delta_{i}} \subseteq \mathcal{A} \cap O_{\beta}$.

Example 3.7. Applied to the quasi-Polish space $\mathcal{P}_{\infty}(\mathbb{N})$ and its basis $\left\{\mathcal{O}_{A} \cap \mathcal{P}_{\infty}(\mathbb{N}) \mid\right.$ $\left.A \in \mathcal{P}_{<\omega}(\mathbb{N})\right\}$ (cf. Examples 2.11, and 2.20), the previous proof gives an approximation relation which is not the containment relation (cf. Example 3.2). Clause $n$ is $\exists j \geq n j \in X$ (i.e. $A_{n}$ is empty). It is an $\mathcal{O}_{A} \cap \mathcal{P}_{\infty}(\mathbb{N})$-clause and it is $\mathcal{O}_{A} \cap \mathcal{P}_{\infty}(\mathbb{N})$-solved if and only if $n \leq \max A$. Then, for $A, B \in \mathcal{P}_{<\omega}(\mathbb{N})$, we have $\mathcal{O}_{A} \cap \mathcal{P}_{\infty}(\mathbb{N}) \ll \mathcal{O}_{B} \cap \mathcal{P}_{\infty}(\mathbb{N})$ if and only if $A \subseteq B$ and $\max (A)<\max (B)$, with the convention: $\max \varnothing=-1$.

The converse of Theorem 3.6 is false.

Proposition 3.8. There exists a Hausdorff approximation space with a countable basis which is not a hereditary approximation space hence neither convergent nor quasi-Polish (by Proposition 3.4 and Theorem 3.6 ).

Proof. Consider the topology $\tau$ on the reals generated by the usual open sets and the set $\mathbb{R} \backslash \mathbb{Q}$ of irrational numbers. A topological basis $\mathcal{B}$ is \{]$a, b[] a,, b[\backslash \mathbb{Q} \mid a<b\}$. Consider the relation $\ll$ on $\mathcal{B}$ defined by the following clauses:

$$
\begin{aligned}
& ] a, b[\ll] c, d[\quad \Longleftrightarrow a<c<d<b \quad \text { (i.e. }[c, d] \subset] a, b[) \\
& ] a, b[\ll] c, d[\backslash \mathbb{Q} \quad \Longleftrightarrow a<c<d<b \\
& ] a, b[\backslash \mathbb{Q} \ll] c, d\left[\backslash \mathbb{Q} \Longleftrightarrow \theta(] a, b[\backslash \mathbb{Q}) \ll_{\text {Baire }} \theta(] c, d[\backslash \mathbb{Q})\right.
\end{aligned}
$$

where $\theta$ is the usual homeomorphism (given by continued fractions) between $\mathbb{R} \backslash \mathbb{Q}$ and the Baire space $\mathbb{N}^{\omega}$ and $\ll_{\text {Baire }}$ is any approximation relation on the family of images by $\theta$ of bounded open real intervals (cf. Propositions 3.5 infra and 3.3 supra). It is easy to check that $\ll$ makes $(\mathbb{R}, \tau)$ an approximation space. However, Theorem 3.14 infra ensures that $(\mathbb{R}, \tau)$ is not a hereditary approximation space since $\mathbb{Q}$ is closed in $(\mathbb{R}, \tau)$ and, as a subspace, has the usual topology induced by $\mathbb{R}$ hence fails the Baire property.

Open Problem 3.9. Suppose a space is $\left(T_{0}\right.$ or $\left.T_{1}\right)$ hereditary approximation space and has a countable topological basis. Is it convergent (hence quasi-Polish)?

\subsection{Characterization as Spaces of Choquet Games}

The next theorem asserts that approximation relations on a topological basis of $X$ are essentially normalized strategies for player Nonempty in the Choquet game $C h(X)$. 
Theorem 3.10. If $X$ is a topological space with a well-orderable topological basis (in particular, if $X$ has a countable basis) then $X$ is an approximation (respectively convergent approximation) space if and only if player Nonempty has a stationary (respectively and convergent) winning strategy in the Choquet game $C h(X)$.

Proof. Suppose « is an approximation relation on some topological basis $\mathcal{B}$ of $X$. Fix some well-ordering of $\mathcal{B}$. Define a stationary strategy $\sigma$ for player Nonempty in the Choquet game $\operatorname{Ch}(X)$ as follows. If $U$ is a non empty set and $x \in U$ then define $\sigma(x, U)=B$ where, letting $C$ be least in $\mathcal{B}$ such that $x \in C \subseteq U$, applying condition (3) of Definition 3.1, $B$ is least in $\mathcal{B}$ such that $C \ll B$ and $x \in B$. If

$$
\left(x_{0}, U_{0}\right), B_{0},\left(x_{1}, U_{1}\right), B_{1}, \ldots
$$

is a play where Nonempty follows this strategy $\sigma$, there are open sets $C_{0}, C_{1}, \ldots$ in $\mathcal{B}$ such that

$$
U_{0} \supseteq C_{0} \ll B_{0} \supseteq U_{1} \supseteq C_{1} \ll B_{1} \ldots
$$

Applying condition (2), we see that $B_{0} \ll B_{1} \ll \ldots$ Condition (4) of Definition 3.1 ensures that $\bigcap_{i \in \mathbb{N}} U_{i}=\bigcap_{i \in \mathbb{N}} B_{i} \neq \varnothing$. Thus, $\sigma$ is a winning strategy for player Nonempty. In case $\ll$ is convergent, condition $\left(4^{+}\right)$ensures that $\sigma$ is convergent.

Conversely, suppose that $\tau$ is a winning stationary strategy for Nonempty in the Choquet game $C h(X)$. In particular, $x \in \tau(x, U) \subseteq U$ for all open set $U$ and $x \in U$. Fix some basis $\mathcal{B}$ of $X$ and define a relation $\ll$ on this basis as follows: for $B, C \in \mathcal{B}$

$$
B \ll C \Longleftrightarrow \exists D \in \mathcal{B} \exists x \in D(B \supseteq D \wedge x \in C \subseteq \tau(x, D)) .
$$

Let us check the four conditions of Definition 3.1. Condition (1) is trivial since $C \subseteq$ $\tau(x, D) \subseteq D \subseteq B$. Condition (2) is obvious from the definition of $\ll$. As for condition (3), if $x \in B$ then $x \in \tau(x, B)$ so that, for any $C \in \mathcal{B}$ such that $x \in C \subseteq \tau(x, B)$, we have $B \ll C$. We now show condition (4). Suppose that $B_{n} \ll B_{n+1}$ for all $n$. Let $D_{n} \in \mathcal{B}, x_{n} \in D_{n}$ be such that $B_{n} \supseteq D_{n}$ and $x_{n} \in B_{n+1} \subseteq \tau\left(x_{n}, D_{n}\right)$. Observe that the infinite sequence

$$
\left(x_{0}, D_{0}\right), \tau\left(x_{0}, D_{0}\right),\left(x_{1}, D_{1}\right), \tau\left(x_{1}, D_{1}\right),\left(x_{2}, D_{2}\right), \tau\left(x_{2}, D_{2}\right), \ldots
$$

is a legal play of the Choquet game $C h(X)$ in which Nonempty follows his winning strategy $\tau$. Since $\tau\left(x_{n}, D_{n}\right) \supseteq B_{n+1} \supseteq D_{n+1} \supseteq \tau\left(x_{n+1}, D_{n+1}\right)$, we have $\bigcap_{n \in \mathbb{N}} B_{n}=\bigcap_{i \in \mathbb{N}} D_{n} \neq \varnothing$. In case $\tau$ is a convergent stationary strategy, it is clear that $\ll$ is a convergent approximation relation.

Remark 3.11. The above proof fails if we consider the Banach-Mazur game in place of the Choquet game: we fail to obtain condition (3) for the relation « associated to $\tau$.

\subsection{Quasi-Polish and Second Countable Convergent Approximation Spaces Coincide}

Theorem 3.12. Quasi-Polish spaces coincide with $T_{0}$ convergent approximation spaces with a countable basis.

Proof. By Theorem 3.6, quasi-Polish spaces are convergent approximation spaces. If $X$ is a convergent approximation space then, using Theorem 3.10. Nonempty has a con- 
vergent winning strategy in the Choquet game $C h(X)$. By de Brecht's result (cf. item 3 of Theorem 2.30), if $X$ is also $T_{0}$ and has a countable basis then it is quasi-Polish.

Putting together Theorems 3.12, 3.10 and 2.35 we can complement Corollary 2.33,

Corollary 3.13. Let $X$ be a $T_{0}$ space with a countable basis. The following conditions are equivalent:

1 Nonempty has a convergent wining strategy in the Choquet game $C h(X)$,

2 Nonempty has a stationary convergent wining strategy in the Choquet game $C h(X)$,

$3 \quad X$ is a quasi-Polish space.

\subsection{The $\boldsymbol{\Pi}_{2}^{0}$ Baire Property in Approximation Spaces}

The Baire property holds in Polish spaces and in compact $T_{2}$ (i.e. Hausdorff) spaces, and it is also true in quasi-Polish spaces (de Brecht 2011): the intersection of countably many dense open sets is dense. It trivially implies that the intersection of countably many dense $\mathbf{G}_{\delta}$ sets is dense. The next result is a formulation of the Baire property for spaces where the $\boldsymbol{\Pi}_{2}^{0}$ and $\mathbf{G}_{\delta}$ classes do not coincide. As a corollary, we see that the classical Baire property on $\omega$-continuous domains and also that on quasi-Polish spaces (cf. Theorem 2.25) can be strengthened from $\mathbf{G}_{\delta}$ to $\boldsymbol{\Pi}_{2}^{0}$.

Theorem 3.14. If $X$ is an approximation space with a well-orderable topological basis (in particular, if $X$ has a countable basis) then it satisfies the Baire property for $\Pi_{2}^{0}(E)$ sets: the intersection of countably many dense $\boldsymbol{\Pi}_{2}^{0}(E)$ sets is dense.

Proof. Using Lemma 3.3, consider a well-orderable basis $\mathcal{B}$ and an approximation relation $\ll$ on $\mathcal{B}$. We fix some well-order on $\mathcal{B}$ and we will speak freely of the least $U$ in $\mathcal{B}$ satisfying a given property. Since $\Pi_{2}^{0}(E)$ sets are intersections of countably many sets in $c o-\mathbf{D}_{2}(E)$ (i.e. unions of an open and a closed sets), and any superset of a dense set is dense, it suffices to prove the Baire property for $c o-\mathbf{D}_{2}$ sets. Suppose $X=\bigcap_{n \in \mathbb{N}} U_{n} \cup F_{n}$ where, for each $n, U_{n}$ is open, $F_{n}$ is closed and $U_{n} \cup F_{n}$ is dense. We prove that $X$ is dense, i.e. $X$ meets $O$ for each $O \in \mathcal{B}$. Fix some $\pi: \mathbb{N} \rightarrow \mathbb{N}$ such that $\pi^{-1}(j)$ is infinite for each $j \in \mathbb{N}$. Fix some $O \in \mathcal{B}$. We inductively define a sequence of basic open sets $\left(O_{n}\right)_{n \in \mathbb{N}}$ such that $O \ll O_{0}$ and $O_{n} \ll O_{n+1}$ for all $n$.

(a) Let $O_{0}$ be the least $W \in \mathcal{B}$ such that $O \ll W$ (there exists such a $W$ : take any element $x$ in $O$ and apply condition (3) of Definition 3.1).

(b) Suppose $n \geq 1, O_{n-1}$ is defined and let $W \in \mathcal{B}$ be least such that $O_{n-1} \ll W$ (as above there exists such a $W$ ). If $U_{\pi(n)}$ contains some $W^{*}$ such that $W \ll W^{*}$ then set $O_{n}=W^{*}$. Otherwise, set $O_{n}=W$. The following Claim finishes the proof of the Theorem.

Claim. The set $\bigcap_{n \in \mathbb{N}} O_{n}$ is non empty and is included in $O \cap\left(\cap_{j \in \mathbb{N}} U_{j} \cup F_{j}\right)$.

Proof of Claim. Since $\ll$ is transitive, $O_{n} \ll O_{n+1}$ holds for all $n$ 's and the non emptiness of $\bigcap_{n \in \mathbb{N}} O_{n}$ is ensured by condition (4) of Definition 3.1. Let $x \in \bigcap_{n \in \mathbb{N}} O_{n}$. By clause (a) we have $O \ll O_{0}$. Thus, $O_{0} \subseteq O$ and $x \in O$. Let $j \in \mathbb{N}$. Suppose $x \notin U_{j}$, we show that $x \in F_{j}$. Since $x \notin U_{j}$ we have $O_{n} \nsubseteq U_{j}$ for all $n \in \mathbb{N}$. Let $n$ be such that $\pi(n)=j$, clause (b) ensures that $O_{n}=W$ (instead of $W^{*}$ ) and 
$\left(^{*}\right) \quad U_{j}$ contains no $V \in \mathcal{B}$ such that $O_{n} \ll V$.

Since $F_{j}$ is closed, to show that $x \in F_{j}$, it suffices to prove that $x$ is adherent to $F_{j}$. Consider $T \in \mathcal{B}$ such that $x \in T$, we have to prove that $T$ meets $F_{j}$. Let $n$ be such that $\pi(n)=j$. Since $U_{j} \cup F_{j}$ is dense and $T \cap O_{n}$ is non empty (it contains $x$ ), there exists $x_{n} \in\left(U_{j} \cup F_{j}\right) \cap T \cap O_{n}$. By way of contradiction, suppose $x_{n}$ is in $U_{j}$. Then let $S \in \mathcal{B}$ be such that $x_{n} \in S \subseteq U_{j} \cap T \cap O_{n}$. Condition (3) yields some $V \in \mathcal{B}$ such that $x_{n} \in V$ and $S \ll V$. Using condition (2) and inclusion $S \subseteq O_{n}$ we have $O_{n} \ll V$. Now, we also have $V \subseteq S \subseteq U_{j}$, contradicting $\left(^{*}\right)$. Thus, $x_{n} \in F_{j}$; hence, $T$ meets $F_{j}$.

Remark 3.15. The fact that every continuous domain $D$ satisfies the $\Pi_{2}^{0}(D)$ Baire property questions on a possible relation with the Lawson topology. Recall that the Lawson topology on a continuous domain is the refinement of the Scott topology such that the complement of the uppercone set $\uparrow x$ is open, for each $x$ in $D$. The Lawson topology is $T_{2}$ and compact, so it satisfies the usual Baire property with $\mathbf{G}_{\delta}$ sets. Also, the Scott $\boldsymbol{\Pi}_{2}^{0}$ class is included in the Lawson class $\mathbf{G}_{\delta}$. However, the $\boldsymbol{\Pi}_{2}^{0}$ Baire property for the Scott topology differs from the usual $\mathbf{G}_{\delta}$ Baire property for the Lawson topology because the notion of dense set is not the same in the two topologies.

\subsection{Hausdorff's Theorem and the $\boldsymbol{\Pi}_{2}^{0}$ Baire Property}

Hausdorff-Kuratowski's theorem establishes that for Polish spaces the inclusion in Proposition 2.9 is an equality: $\bigcup_{\alpha<\omega_{1}} \mathbf{D}_{\alpha}\left(\boldsymbol{\Sigma}_{\beta}^{0}(E)\right)=\boldsymbol{\Delta}_{\beta+1}^{0}(E)$. One of the properties of Polish spaces used in the proof of this result is the classical Baire property. Matthew de Brecht (2011) has proved that Hausdorff-Kuratowski's theorem holds for quasi-Polish spaces. Our next theorem gives conditions of approximaton spaces to establish the case $\beta=1$ i.e., Hausdorff's theorem $\boldsymbol{\Delta}_{2}^{0}(E)=\bigcup_{\alpha<\omega_{1}} \mathbf{D}_{\alpha}(E)$. The theorem pinpoints some topological properties that suffice to prove this equality. These properties are true in second countable hereditary approximation spaces. A similar result was obtained in (Tang 1981) for the Scott domain $\mathcal{P}(\mathbb{N})$. Using a different proof Selivanov (2005) proved it for $\omega$-algebraic domains.

Theorem 3.16. Let $E$ be a topological space satisfying the following properties.

(i) There exists a countable basis of open sets.

(ii) Every closed subspace $F$ of $E$ satisfies the $\Pi_{2}^{0}(E)$-Baire property: the intersection of countably many $\Pi_{2}^{0}(E)$ subsets of $F$ which are dense in $F$ is also dense in $F$.

Then, $\bigcup_{\alpha<\omega_{1}} \mathbf{D}_{\alpha}(E)=\Delta_{2}^{0}(E)$.

Proof. The $\subseteq$ inclusion is Proposition 2.9. For the $\supseteq$ inclusion, we follow, mutatis mutandis, Hausdorff's original proof with residues and adjoins, as exposed in (Kechris 1995), Theorem 22.27 pages $176-177$. For any subset $A \subseteq E$, we define by transfinite recursion a family $\left(F_{\alpha}\right)_{\alpha<\omega_{1}}$ :

$$
F_{0}=E, F_{2 \alpha+1}=\overline{A \cap F_{2 \alpha}}, F_{2 \alpha+2}=\overline{(E \backslash A) \cap F_{2 \alpha+1}}, F_{\lambda}=\bigcap_{\alpha<\lambda} F_{\alpha} \quad \text { if } \lambda \text { is limit. }
$$

Applying property (i), let $\left(O_{n}\right)_{n \in \mathbb{N}}$ be a countable basis of open sets of $E$. The $F_{\alpha}$ 's are a decreasing sequence of closed sets. If $F_{\xi+1}=F_{\xi}$ then $F_{\alpha}=F_{\xi}$ for all $\alpha \geq \xi$. If $F_{\alpha+1}$ is a 
strict subset of $F_{\alpha}$ then there is some $O_{n}$ which meets $F_{\alpha}$ but not $F_{\alpha+1}$. Since there are countably many $O_{n}$ 's, this implies that there is some countable $\theta$ such that $F_{\theta}=F_{\alpha}$ for all $\alpha \geq \theta$. We shall consider the least even such $\theta$.

Claim. If $A$ is $\boldsymbol{\Delta}_{2}^{0}(E)$ then $F_{\theta}=\varnothing$.

Proof of Claim. Suppose $F_{\theta} \neq \varnothing$. Applying property (ii), the subspace $F_{\theta}$ is in $E$ hence it satisfies the Baire property. Since $F_{\theta}=F_{\theta+2}$, we have $F_{\theta}=\overline{A \cap F_{\theta}}=\overline{(E \backslash A) \cap F_{\theta}}$. Thus, arguing in the subspace $F_{\theta}$, the sets $A \cap F_{\theta}$ and $(E \backslash A) \cap F_{\theta}$ are $\Pi_{2}^{0}\left(F_{\theta}\right)$ dense subsets of $F_{\theta}$. Since they are disjoint, this contradicts property (ii) in $F_{\theta}$.

Letting $\theta=2 \zeta$ and $B=\bigcup_{\alpha<\zeta} F_{2 \alpha+1} \backslash F_{2 \alpha+2}$, we claim that $A=B$. Indeed, suppose $x \in A$ and let $\eta$ be least such that $x \notin F_{\eta}$. The inductive definition of the $F_{\alpha}$ 's ensures that $\eta=2 \alpha+2$ for some $\alpha$. Therefore, $x \in F_{2 \alpha+1} \backslash F_{2 \alpha+2}$ hence $x \in B$. Similarly, if $x \notin A$ and $\eta$ is least such that $x \notin F_{\eta}$ then $\eta=2 \alpha+1$ for some $\alpha$. Thus, $x \in F_{2 \alpha} \backslash F_{2 \alpha+1}$ hence $x \notin B$. Observe that, for $\lambda$ limit, we have $\left(E \backslash F_{\lambda}\right) \backslash \bigcup_{\alpha<\lambda}\left(E \backslash F_{\alpha}\right)=\varnothing$. Thus, $A=B=\bigcup_{\alpha<\zeta}\left(E \backslash F_{2 \alpha+2}\right) \backslash\left(E \backslash F_{2 \alpha+1}\right)=D_{\theta+1}\left(\left(E \backslash F_{\alpha}\right)_{\alpha \leq \theta}\right)$ hence $A$ is in $\mathbf{D}_{\theta+1}(E)$.

Corollary 3.17. Equality $\bigcup_{\alpha<\omega_{1}} \mathbf{D}_{\alpha}(E)=\boldsymbol{\Delta}_{2}^{0}(E)$ holds in any hereditary approximation space having a countable basis.

Open Problem 3.18. What topological conditions ensure higher levels of HausdorffKuratowski theorem?

\section{The Hausdorff Hierarchy in Continuous Domains}

Selivanov made a fine analysis of the Hausdorff difference hierarchy in $\omega$-algebraic domains and he proved Hausdorff's theorem for these spaces (Selivanov 2005). He also showed the non existence of ambiguous sets in the Hausdorff hierarchy, provided there is a least element in the $\omega$-algebraic domain. As stated in (Selivanov 2008, Theorem 3.4), the methods in his paper of 2005 can be pushed from $\omega$-algebraic to $\omega$-continuous domains.

In this section we reconsider the question of the non existence of ambiguous sets in the Hausdorff hierarchy for continuous domains (possibly not $\omega$-continuous). We prove that the hypothesis of a least element considered by Selivanov can be removed for successor levels of the Hausdorff hierarchy, but not for limit levels. Although this improvement is a modest addition to Selivanov's result (proofs being, mutatis mutandis, the same), it requires to use the machinery of well-founded alternating trees developed in (Selivanov 2005). The extension from $\omega$-continuous domains to continuous domains led us to consider possibly non countable alternating trees. In fact, the countability of the domain basis is useful only to ensure that the tree is countable, hence the rank of an alternating tree is countable. But this hypothesis appears directly as an assumption on the ordinal $\alpha$ in Theorems 4.7 and 4.8 .

\subsection{Alternating Trees}

First, we recall some simple notions about possibly uncountable trees.

\section{Definition 4.1.}


1 A tree is any non empty set of finite sequences closed under prefix. The root of a tree is the empty sequence nil. If $\sigma$ is in a tree $T$ we let $T_{\sigma}$ be the tree $\{\tau \mid \sigma \tau \in T\}$.

2 A tree $T$ is well-founded if it has no infinite branch. The ranks of the elements in a well-founded tree $T$ are defined inductively: $\operatorname{rank}_{T}(\sigma)=\sup \left\{\operatorname{rank}_{T}(\sigma n)+1 \mid \sigma n \in T\right\}$ (convention for leaves of $T: \sup \varnothing=0$ ). The rank of $T$ is that of its root.

3 An alternating tree is a map $f: T \rightarrow\{0,1\}$ such that $T$ is a tree and $f(\tau) \neq f(\sigma)$ whenever $\sigma$ is a son of $\tau$ (i.e. $\tau$ is a prefix of $\sigma$ and the length of $\sigma$ is the successor of that of $\tau)$. We say $f$ is $\varepsilon$-alternating if $f(n i l)=\varepsilon$.

4 An embedding of an alternating tree $g: S \rightarrow\{0,1\}$ into an alternating tree $f: T \rightarrow$ $\{0,1\}$ is a monotone increasing (with respect to the prefix ordering on finite sequences) injective map $\theta: S \rightarrow T$ such that $g(\sigma)=f(\theta(\sigma))$. We write $g \leq f$.

The next basic result is taken from (Selivanov 2005), Lemma 3.6 page 48.

Lemma 4.2. Let $f: T \rightarrow\{0,1\}$ be a well-founded alternating tree with rank $\alpha$. For every $\beta<\alpha$ and every $\varepsilon \in\{0,1\}$, there exists an $\varepsilon$-alternating tree $g_{\varepsilon} \leq f$ with rank $\beta$.

Proof. We reproduce Selivanov's proof. Argue by induction on $\alpha$. If $\alpha$ is finite then take a branch of length $\alpha$ and remove an appropriate tail and/or the root. If $\alpha$ is limit then, for some $x, T_{(x)}$ (cf. Definition 4.1) has rank $\gamma$ such that $\beta<\gamma<\alpha$. Use the induction hypothesis with $f_{(x)}: T_{(x)} \rightarrow\{0,1\}$ such that $f_{(x)}(\sigma)=f(x \sigma)$. Finally, suppose $\alpha=\lambda+m+1$ with $\lambda$ limit and $m \in \mathbb{N}$. If $\beta<\lambda+m$ then let $x$ such that $T_{(x)}$ has rank $\lambda+m$ and use the induction hypothesis. Suppose now $\beta=\lambda+m$. Let $s$ with length $m+1$ such that $T_{s}$ has rank $\lambda$. Let $\eta$ be the cofinality of $\lambda$ and let $\left(\lambda_{\xi}\right)_{\xi<\eta}$ be strictly increasing with supremum $\lambda$ and $x_{\xi}$ be such that $T_{s x_{\xi}}$ has rank $\lambda_{\xi}$. Let $g_{\xi, \delta} \leq f_{s x_{\xi+1}}$ for $\delta \in\{0,1\}$ be $\delta$-alternating with rank $\lambda_{\xi}$. Set $g_{\varepsilon}\left(0^{p}\right)=f(s \uparrow p)$ for $p \leq m$ and $g_{\varepsilon}\left(0^{m} \xi \sigma\right)=g_{\xi, \delta}(\sigma)$ where $\delta$ is chosen so as to get alternation from $0^{m}$ to $0^{m} \xi$.

\subsection{Alternating Trees and the Hausdorff Hierarchy}

The following notion plays the role of intervals $[p, x]$ in (Selivanov 2005).

Definition 4.3. Let $b, x$ be elements of the domain $D$. We let $\llbracket b, x]=\{y \mid b \ll y \leq x\}$.

Proposition 4.4. Let $\left(D\right.$, ㄷ) be a dcpo and $A \in \boldsymbol{\Sigma}_{2}^{0}(D)$. If $\left(x_{i}\right)_{i \in I}$ is a directed system with supremum in $A$ then $x_{i} \in A$ for all $i$ large enough. In particular, if $D$ is a continuous domain with basis $B \subseteq D$ and $x \in A$ then there exists $b \in B$ such that $b \ll x$ and $\llbracket b, x] \subseteq A$.

Proof. Let $A=\cup_{n \in \mathbb{N}} U_{n} \backslash V_{n}$ where $U_{n}, V_{n}$ are open. Let $x=\sqcup_{i} x_{i}$. Since $x \in U_{n}$ and $U_{n}$ is Scott open, the $x_{i}$ 's are in $U_{n}$ for $i$ large enough. Since $x \notin V_{n}$ and $V_{n}$ is an upset, no $x_{i}$ is in $V_{n}$. Thus, the $x_{i}$ 's are in $U_{n} \backslash V_{n}$ hence in $A$ for $i$ large enough.

To keep the presentation self-contained, we prove the extensions to continuous domains of Theorems 3.10 and 3.14 in (Selivanov 2005). Proofs are almost the same: compact elements are replaced by elements of some fixed basis $B$ and the countability of the basis (which ensures the countability of the rank of alternating $B$-trees, cf. Definition 4.5) is replaced by the assumed countability of the considered ordinal $\alpha$. 
Definition 4.5. Let $(D, \sqsubseteq)$ be a dcpo and $B \subseteq D$. If $A$ is any subset of $D$ we let $\chi_{A}: A \rightarrow\{0,1\}$ be the characteristic function of $A$ (which takes value 1 on $A$ ).

1 A $B$-tree is a map $f: T \rightarrow B$ where $T$ is a tree.

2 A $B$-tree $f: T \rightarrow B$ is $(A, \varepsilon)$-alternating if the tree $\chi_{A} \circ f: T \rightarrow\{0,1\}$ is $\varepsilon$-alternating in the sense of Definition 4.1 i.e. $f(\sigma n) \in A \Leftrightarrow f(\sigma) \notin A$ for all $n \in \mathbb{N}$ and $\sigma, \sigma n \in T$.

3 Let $\leq$ be 5 or $\ll$. A $B$-tree $f$ is $\leq$-increasing if $f(\sigma) \leq f(\sigma n)$ for all $\sigma, \sigma n \in T$.

Proposition 4.6. Let $(D, \sqsubseteq)$ be a dcpo and $B \subseteq D$. If $A$ is $\boldsymbol{\Delta}_{2}^{0}(D)$ then every $A$ alternating ㄷ-increasing $B$-tree $f: T \rightarrow B$ is well-founded.

Proof. Let $\left(n_{i}\right)_{i \in \mathbb{N}}$ be an infinite branch of $f$. The $f\left(n_{0} \ldots n_{i}\right)$ 's are ㄷ-increasing. Let $x$ be their supremum. Suppose $x \in A$. Proposition 4.4 establishes that $f\left(n_{0} \ldots n_{i}\right)$ is in $A$ for all $i$ large enough, contradicting alternation of $f$. Idem if $x \notin A$.

The next result is a slight variant of (Selivanov 2005, Proposition 3.8 and Theorem 3.10).

Theorem 4.7. Let $(D, \sqsubseteq)$ be a dcpo, $B \subseteq D, A \in \boldsymbol{\Delta}_{2}^{0}(D)$ and $0<\alpha<\omega_{1}$.

1 If $A \in \mathbf{D}_{\alpha}(D)$ then there is no $\leq$-increasing $(A, 1)$-alternating $B$-tree with rank $\alpha$.

2 If $D$ is a continuous domain with basis $B$, the following conditions are equivalent.

i. $A \in \mathbf{D}_{\alpha}(D)$,

ii. there is no ㄷ-increasing $(A, 1)$-alternating $B$-tree with rank $\alpha$.

iii. there is no $\ll$-increasing $(A, 1)$-alternating $B$-tree with rank $\alpha$.

Proof. 1. We argue by induction on $\alpha$. Let $A=D_{\alpha}\left(\left(A_{\beta}\right)_{\beta<\alpha}\right)$ where the $A_{\beta}$ 's are a monotone increasing $\alpha$-sequence of open sets. By way of contradiction, suppose there exists an increasing $(A, 1)$-alternating $B$-tree $f: T \rightarrow B$ with rank $\alpha$. Since $f(n i l) \in A$ we have $f($ nil $) \in A_{\beta} \backslash \cup_{\gamma<\beta} A_{\gamma}$ for some $\beta<\alpha, \beta \psi \alpha$. Since $f(n i l) \leq f(\sigma)$ for all $\sigma \in T$ and $A_{\beta}$ is open (hence, an upset), we see that the range of $f$ is included in $A_{\beta}$. If $(n) \in T$ then $f((n)) \notin A$ hence $f((n)) \in A_{\beta} \backslash A \subseteq \cup_{\gamma<\beta} A_{\gamma}$. Since $\cup_{\gamma<\beta} A_{\gamma}$ is open (hence, an upset) we see that $f(\sigma) \in \bigcup_{\gamma<\beta} A_{\gamma}$ for all $\sigma \in T, \sigma \neq n i l$. Let $A^{-}=D_{\alpha}\left(\left(A_{\gamma}\right)_{\gamma<\beta}\right)$. Then $f$ is an $\left(A^{-}, 0\right)$-alternating $B$-tree. Now, $f$ has rank $\alpha$ and Lemma 4.2 implies that there exists $g \leq f$ which is an $\left(A^{-}, 1\right)$-alternating $B$-tree with $\operatorname{rank} \beta$. Since $A^{-}$is in $\mathbf{D}_{\beta}$, the inductive hypothesis is contradicted.

2. Since $i \Rightarrow i i$ is item 1 and $i i \Rightarrow i i i$ is trivial, it remains to prove $i i i \Rightarrow i$. For each $b \in B$, let $S_{b}$ be the family of finite sequences $\left(b, b_{1}, \ldots, b_{k}\right)$ of elements of $B$ satisfying the following conditions:

$-b \ll b_{1} \ll \ldots \ll b_{k}$,

- $b_{i} \in A \Leftrightarrow b_{i+1} \notin A$ for all $0 \leq i<k$ (with $b_{0}=b$ ).

Fix some bijection $\theta$ between $B$ and an initial segment of $\mathbb{N}$. Applying $\theta$, transform $S_{b}$ into an $A$-alternating $B$-tree $f_{b}: T_{b} \rightarrow B$ such that

$$
T_{b}=\{n i l\} \cup\left\{\left(\theta\left(b_{1}\right), \ldots, \theta\left(b_{k}\right)\right) \mid\left(b, b_{1}, \ldots, b_{k}\right) \in S_{b}\right\}
$$

and $f_{b}(n i l)=b, f_{b}\left(\left(\theta\left(b_{1}\right), \ldots, \theta\left(b_{k}\right)\right)\right)=b_{k}$. Since $A$ is assumed to be $\boldsymbol{\Delta}_{2}^{0}(D)$, Proposition 4.6 ensures that $f_{b}$ is well-founded. Suppose there is no $(A, 1)$-alternating $B$-tree 
with rank $\alpha$. Then $f_{b}$ has rank $\leq \alpha$ if $b \notin A$ and rank $<\alpha$ if $b \in A$. For $\beta<\alpha$ define the open sets

$$
A_{\beta}=\bigcup\left\{\uparrow f_{b}(\sigma) \mid b \in B, \operatorname{rank}_{T_{b}}(\sigma) \leq \beta, f_{b}(\sigma) \in A \Leftrightarrow \beta \psi \alpha\right\} .
$$

To conclude we prove that $A=\mathbf{D}_{\alpha}\left(\left(A_{\beta}\right)_{\beta<\alpha}\right)$. First, we show that $A \subseteq \bigcup_{\beta<\alpha} A_{\beta}$. Suppose $x \in A$. Applying Proposition 4.4 we get $\llbracket b, x] \subseteq A$ for some $b \ll x, b \in B$. Since $b \in A$, we have $\operatorname{rank}\left(T_{b}\right)<\alpha$. A fortiori, $\operatorname{rank}\left(T_{b}\right) \leq \beta$ with $\beta<\alpha$ and $\beta \psi \alpha$. Since $f_{b}($ nil $)=b$ we have $\uparrow b \subseteq A_{\beta}$ hence $x \in A_{\beta}$. For $\beta<\alpha$, let $A_{\beta}^{\prime}=A_{\beta} \backslash \bigcup_{\gamma<\beta} A_{\gamma}$. Since $A \subseteq \bigcup_{\beta<\alpha} A_{\beta}$, to show $A=\mathbf{D}_{\alpha}\left(\left(A_{\beta}\right)_{\beta<\alpha}\right)$ it suffices to prove that $\beta \psi \alpha \Rightarrow A_{\beta}^{\prime} \subseteq A$ and $\beta \sim \alpha \Rightarrow A_{\beta}^{\prime} \subseteq D \backslash A$.

Case $\beta \nmid \alpha$. By way of contradiction, suppose $A_{\beta}^{\prime} \nsubseteq A$ and let $x \in A_{\beta}^{\prime} \backslash A$. By Proposition 4.4 we have $\llbracket c, x] \subseteq D \backslash A$ for some $c \ll x, c \in B$. Now, since $x \in A_{\beta}$, there exist $b \in B$ and $\sigma \in T_{b}$ such that $x \in \uparrow f_{b}(\sigma), \operatorname{rank}_{T_{b}}(\sigma) \leq \beta, f_{b}(\sigma) \in A$. Since $c, f_{b}(\sigma) \ll x$, the interpolation property gives an $e \in B$ such that $c, f_{b}(\sigma) \ll e \ll x$. Since $\left.e \in \llbracket c, x\right]$ we have $e \notin A$. Let $\sigma=\left(\theta\left(b_{1}\right), \ldots, \theta\left(b_{k}\right)\right)$ where $\left(b, b_{1}, \ldots, b_{k}\right) \in S_{b}$. Since $b_{k}=f_{b}(\sigma) \in A, f_{b}(\sigma) \ll e$ and $e \notin A$, the sequence $\left(b, b_{1}, \ldots, b_{k}, e\right) \in S_{b}$. Hence $\sigma \theta(e) \in T_{b}$. Now, $\operatorname{rank}(\sigma \theta(e))<\operatorname{rank}(\sigma) \leq \beta$. Since $\beta \psi \alpha$, there is some $\gamma<\beta$ such that $\gamma \sim \alpha$ and $\operatorname{rank}(\sigma \theta(e)) \leq \gamma$. Summing up, we have $\uparrow e \subseteq A_{\gamma}$. Since $e \ll x$ we get $x \in A_{\gamma}$ which contradicts $x \in A_{\beta}^{\prime}$.

Case $\beta \sim \alpha$. The proof that $A_{\beta}^{\prime} \subseteq D \backslash A$ is similar.

\subsection{Ambiguous Sets in the Hausdorff Hierarchy}

We now come to the question of whether there are ambiguous sets in the Hausdorff hierarchy. Item 1 of the next Theorem was obtained by Selivanov (2005) for $\omega$-algebraic domains.

Theorem 4.8. Let $D$ be a continuous domain and $0 \leq \alpha<\omega_{1}$.

1 If $D$ has a least element $\perp$ then $\mathbf{D}_{\alpha}(D) \cap c o-\mathbf{D}_{\alpha}(D)=\bigcup_{\beta<\alpha} \mathbf{D}_{\beta}(D) \cup c o-\mathbf{D}_{\beta}(D)$.

$2 \mathbf{D}_{\alpha+1}(D) \cap c o-\mathbf{D}_{\alpha+1}(D)=\bigcup_{\beta \leq \alpha} \mathbf{D}_{\beta}(D) \cup c o-\mathbf{D}_{\beta}(D)$ for all $1 \leq \alpha<\omega_{1}$.

3 In general, equality $\mathbf{D}_{\alpha}(D) \cap c o-\mathbf{D}_{\alpha}(D)=\bigcup_{\beta<\alpha} \mathbf{D}_{\beta}(D) \cup c o-\mathbf{D}_{\beta}(D)$ fails for $\alpha=1$ and for $\alpha$ limit.

Proof. 1. Inclusion right to left comes from Proposition 2.6. Inclusion left to right is proved by induction on $\alpha$. Case $\alpha=0$ is trivial since both members are empty.

Suppose $\alpha=\beta+1$ and $A \in \mathbf{D}_{\alpha}(D) \cap c o-\mathbf{D}_{\alpha}(D)$. Towards a contradiction, suppose $A \notin \mathbf{D}_{\beta}(D) \cup c o-\mathbf{D}_{\beta}(D)$, i.e. neither $A$ nor $D \backslash A$ is in $\notin \mathbf{D}_{\beta}(D)$. Theorem 4.7 proves the existence, for $\varepsilon=0,1$, of an increasing $(A, \varepsilon)$-alternating $B$-tree $f_{\varepsilon}: T_{\varepsilon} \rightarrow B$ with rank $\beta$. Observe that every domain basis contains $\perp$, hence $\perp \in B$. Let $\tau=0$ if $\perp \in A$ and $\tau=1$ otherwise, so that $f_{\tau}(n i l) \neq 1$. Let $S=\left\{0 \sigma \mid \sigma \in T_{\tau}\right\}$ and $g: S \rightarrow B$ be such that $g(n i l)=\perp$ and $g(0 \sigma)=f_{\varepsilon}(\sigma)$. Then $g$ is an increasing $(A, 1-\tau)$-alternating $B$-tree with rank $\beta+1=\alpha$. Applying again Theorem 4.7, if $\tau=0$ this contradicts $A \in \mathbf{D}_{\alpha}(D)$, if $\tau=1$ this contradicts $A \in c o-\mathbf{D}_{\alpha}(D)$.

Suppose now $\alpha$ is a limit ordinal and $\left(\alpha_{n}\right)_{n \in \mathbb{N}}$ is increasing with $\alpha$ as supremum. Towards a contradiction, suppose $A \notin \cup_{\beta<\alpha} \mathbf{D}_{\beta}(D) \cup c o-\mathbf{D}_{\beta}(D)$. Then, for every $n$ and $\varepsilon=0,1$, there is a monotone increasing $(A, \varepsilon)$-alternating $B$-tree $f_{n, \varepsilon}: T_{n, \varepsilon} \rightarrow B$ with rank $\alpha_{n}$. Let $\tau \in\{0,1\}$ be as above. Set $S=\left\{n \sigma \mid \sigma \in T_{n, \varepsilon}\right\}$ and $g: S \rightarrow B$ be such 
that $g(n i l)=\perp$ and $g(n \sigma)=f_{n, \varepsilon}(\sigma)$. Then $g$ is a monotone increasing $(A, \tau)$-alternating $B$-tree with rank $\alpha$. As above, this gives a contradiction.

2. Let $A=D_{\alpha+1}\left(\left(A_{\beta}\right)_{\beta \leq \alpha}\right)$ and $D \backslash A=D_{\alpha+1}\left(\left(E_{\beta}\right)_{\beta \leq \alpha}\right)$ where the $A_{\beta}, E_{\beta}$ 's are $\alpha+1$ increasing sequences of open sets. Set $D^{+}=D \cup\left\{\perp_{\alpha}\right\}$ and extend the order of $D$ by setting $\perp_{\alpha}<x$ for all $x \in A_{\alpha}$. Set $A^{+}=A \cup\left\{\perp_{\alpha}\right\}$. Then, in $D^{+}$, we have $A^{+}=D_{\alpha+1}\left(\left(A_{\beta}^{*}\right)_{\beta \leq \alpha}\right)$ where $A_{\alpha}^{*}=A_{\alpha} \cup\left\{\perp_{\alpha}\right\}$ and $A_{\beta}^{*}=A_{\beta}$ for $\beta<\alpha$. Also, $D^{+} \backslash A^{+}=D \backslash A$. Observe that the $A_{\beta}^{*}$ 's and $E_{\beta}$ 's are open in $D^{+}$. Thus, $A^{+}$is ambiguous at level $\alpha+1$ in $D^{+}$. Though $\perp_{\alpha}$ is not a least element in $D^{+}$, it is smaller than $A_{\alpha}$ hence smaller than all elements labeling an $(A, 1)$-alternating $B$-tree with rank $\alpha$. Arguing as in item 1 , we see that $A^{+}$must have level at most $\alpha$ in $D^{+}$. If $D^{+} \backslash A^{+}=D \backslash A$ has level $\alpha$ in $D^{+}$then $D \backslash A$ is obtained via open sets not containing $\perp_{\alpha}$ hence $D \backslash A$ has level $\alpha$ in $D$. Suppose now that $A^{+}$has level $\alpha$ in $D^{+}$. Then $A^{+}=D_{\alpha}\left(\left(C_{\delta}\right)_{\delta<\alpha}\right)$ where the $C_{\delta}$ 's are open in $D^{+}$. Since $\perp_{\alpha} \in A^{+}$there is some $\beta<\alpha$ with parity different from $\alpha$ such that $\perp_{\alpha} \in C_{\beta}$ hence $C_{\beta} \supset \bigcup_{\delta<\alpha} A_{\delta}$. This yields $A=D_{\alpha}\left(\left(C_{\gamma}^{-}\right)_{\gamma<\alpha}\right)$ where $C_{\gamma}^{-}=C_{\gamma}$ for $\gamma<\beta$ and $C_{\gamma}^{-}=C_{\gamma} \cap D$ for $\gamma \geq \beta$. So, $A$ has level $\beta$ in $D$. Finally, observe that the argument breaks down if $\alpha=0$.

3. In $\mathbf{2}^{\leq \omega}$, the set $0 \mathbf{2}^{\leq \omega}$ is in $\mathbf{D}_{1}(D) \cap c o-\mathbf{D}_{1}(D)$ but not in $\mathbf{D}_{0}(D) \cap c o-\mathbf{D}_{0}(D)$. Let $\alpha$ be a countable limit ordinal. Let $\left(a_{\beta}\right)_{\beta<\alpha}$ be a strictly decreasing $\alpha$-sequence of reals with no lower bound and which is continuous: $a_{\lambda}=\inf _{\delta<\lambda} a_{\delta}$ for all limit $\lambda<\alpha$. In $\overrightarrow{\mathbb{R}}$ (cf. Example 2.11), consider the set $A=\mathbf{D}_{\alpha}\left(\left(A_{\beta}\right)_{\beta<\alpha}\right)$ where $\left.\left.A_{\beta}=\right] a_{\beta},+\infty\right]$. Then $\overrightarrow{\mathbb{R}} \backslash A=\mathbf{D}_{\alpha}\left(\left(A_{\beta}^{*}\right)_{\beta<\alpha}\right)$ where $A_{0}^{*}=\varnothing$ and $A_{\beta+1}^{*}=A_{\beta}$ and $A_{\lambda}^{*}=A_{\lambda}$ for $\lambda$ limit. Thus, $A$ is ambiguous at level $\alpha$. It is easy to check that $A$ is not in a lesser level.

\section{Effective Borel and Hausdorff Hierarchies}

\subsection{Effective Topological Spaces}

The first step to deal with the effective Borel hierarchy is the definition of effective topological space. We follow Weihrauch's book (2000), Definition 3.2.1, page 63.

Definition 5.1. An effective topological space is a pair $\left(E,\left(O_{n}\right)_{n \in \mathbb{N}}\right)$ where $E$ is a topological space admitting a countable basis and $\left(O_{n}\right)_{n \in \mathbb{N}}$ is an enumeration (not necessarily injective) of some topological basis of $E$.

Remark 5.2. For the notion of computable topological space, one also requires that the equivalence relation $\left\{(m, n) \mid O_{m}=O_{n}\right\}$ be computably enumerable. For instance, this is the case if $n \mapsto O_{n}$ is injective, which is usually true. We shall not need this notion.

Definition 5.3. An effective approximation space is a triple $\left(E,\left(O_{n}\right)_{n \in \mathbb{N}}, \ll\right)$ such that $\left(O_{n}\right)_{n \in \mathbb{N}}$ enumerates a topological basis $\mathcal{B}$, the relation $\left\{(i, j) \mid O_{i} \ll O_{j}\right\}$ is computably enumerable and $\ll$ is an approximation relation on $\mathcal{B}$ (cf. Definition 3.1).

\subsection{Effective w-Continuous Domains}

Definition 5.4. An $\omega$-continuous domain is effective if it admits a basis $B=\left\{b_{n} \mid n \in \mathbb{N}\right\}$ such that $\left\{(i, j) \mid b_{j} \ll b_{i}\right\}$ is computably enumerable. 
Example 5.5. $\left(\mathcal{P}_{\infty}(\mathbb{N}), \subseteq\right)$ is not a continuous domain (cf. Example 3.7) but it is an effective approximation space. Other spaces in Example 2.11 are effective $\omega$-continuous domains.

Proposition 5.6. Every effective $\omega$-continuous domain is an effective approximation space (hence an effective topological space).

Proof. Immediate from the proof of Proposition 3.5 .

\subsection{Borel Codes}

There are several ways to code Borel sets by elements in the Baire space $\mathbb{N}^{\omega}$, cf. (Moschovakis 1979/2009) $\S 3 \mathrm{H}$ and $7 \mathrm{~B}$, (Marker 2002) $\S 7$. We choose a coding adapted to the context of effective topological spaces.

Definition 5.7. Let $\left(E,\left(O_{n}\right)_{n \in \mathbb{N}}\right)$ be an effective topological space. We code Borel sets by well-founded trees (cf. Definition 4.1). To any $\sigma$ in a well-founded tree $T$ we attach a Borel subset $[\sigma]$ of $E$ by induction on the rank.

(i) If $\operatorname{rank}_{T}(n i l)=0$ (i.e. the tree $T$ is reduced to its root) then $[\mathrm{nil}]_{T}=\varnothing$,

(ii) If $\operatorname{rank}_{T}(\sigma)=0$ and $\sigma \neq n i l$ and $\sigma$ has last element $n$ then $[\sigma]_{T}=O_{n}$,

(iii) If $\operatorname{rank}_{T}(\sigma)=1$ then $[\sigma]_{T}=\bigcup_{n \in \mathbb{N}: \sigma n \in T} O_{n}$.

(iv) If $\operatorname{rank}_{T}(\sigma) \geq 2$ then $[\sigma]_{T}=\cup_{n \in \mathbb{N}: \sigma^{\sim} 2 n, \sigma^{\sim} 2 n+1 \in T}\left[\sigma^{\wedge} 2 n\right]_{T} \backslash\left[\sigma^{\wedge} 2 n+1\right]_{T}$.

The $\Sigma$-Borel and $\Pi$-Borel sets coded by $T$ are $[T]_{\Sigma}=[n i l]_{T}$ and $[T]_{\Pi}=E \backslash[n i l]_{T}$.

Observing that the above inductive definition of $[\sigma]_{T}$ follows exactly that of the Borel hierarchy, a straightforward induction on the ordinal $\alpha$ shows the following result.

Proposition 5.8. Let $\left(E,\left(O_{n}\right)_{n \in \mathbb{N}}\right)$ be an effective topological space. A subset of $E$ is in $\boldsymbol{\Sigma}_{\alpha}^{0}(E)$ (respectively $\Pi_{\alpha}^{0}(E)$ ) if and only if it is of the form $[T]_{\Sigma}$ (respectively $[T]_{\Pi}$ ) for some well-founded tree with rank at most $\alpha$.

\subsection{The Effective Borel Hierarchy}

Borel codes lead to a definition of the effective Borel hierarchy. First, we recall some classical results about computable ordinals which imply that there is a huge latitude to represent them: from computability with very low resource complexity up to hyperarithmeticity.

Proposition 5.9. There exists an ordinal $\omega_{1}^{C K}$ (the Church-Kleene ordinal) such that, for every countable ordinal $\alpha$, the following properties are equivalent.

(i) $\alpha<\omega_{1}^{C K}$,

(ii) $\alpha$ is the rank of some computable well-founded tree,

(iii) $\alpha$ is the rank of some hyperarithmetical (i.e. $\Delta_{1}^{1}$ ) well-founded tree (Spector 1955),

(iv) $\alpha$ is the order type of some computable linear order on $\mathbb{N}$, i.e. $\alpha$ is computable,

(v) $\alpha$ is the order type of some hyperarithmetical linear order on $\mathbb{N}$, i.e. $\alpha$ is $\Delta_{1}^{1}$, 
(vi) $\alpha$ is the order type of some linear order on $\mathbb{N}$ which is computable in real time and logarithmic space (Dehornoy 1986, Grigorieff 1990).

Moreover, in (iv-vi), one can suppose that, for each $n \in \mathbb{N}$, the set of elements with rank exactly $n$ and that with ranks in $\left\{\omega \alpha+n \mid \alpha<\omega_{1}^{C K}\right\}$ are computable.

Remark 5.10. The last assertion in Proposition 5.9 is a simple trick in (Ershov 1968). If $(\mathbb{N}, R)$ has type $\alpha$ then the lexicographic product of $(\mathbb{N}, R)$ and $(\mathbb{N},<)$ has type $\omega \alpha$ and the (computable) set $\mathbb{N} \times\{n\}$ consists of all elements of rank $\equiv n \bmod \omega$.

The definition of the effective version of Borel hierarchy for countably based spaces, and the basic properties appeared previously in (Selivanov 2008).

Definition 5.11 (Effective Borel hierarchy). Let $\left(E,\left(O_{n}\right)_{n \in \mathbb{N}}\right)$ be an effective topological space and $\alpha$ an ordinal such that $1 \leq \alpha<\omega_{1}^{C K}$.

1 The effective Borel classes $\Sigma_{\alpha}^{0}(E), \Pi_{\alpha}^{0}(E), \Delta_{\alpha}^{0}(E)$, are defined as follows:

- A set is in the class $\Sigma_{\alpha}^{0}(E)$ (respectively $\Pi_{\alpha}^{0}(E)$ ) if and only if it is of the form $[T]_{\Sigma}$ (respectively $[T]_{\Pi}$ ) for some well-founded tree $T$ with rank at most $\alpha$ such that both $T$ and the rank order relation on $T$ (i.e. $\left.\left\{(s, t) \in T \times T \mid \operatorname{rank}_{T}(s) \leq \operatorname{rank}_{T}(t)\right\}\right)$ are computable.

— The class $\Delta_{\alpha}^{0}(E)=\Sigma_{\alpha}^{0}(E) \cap \Pi_{\alpha}^{0}(E)$.

2 A sequence $\left(X_{n}\right)_{n \in \mathbb{N}}$ of subsets of $E$ is uniformly $\Sigma_{\alpha}^{0}(E)$ if there exists a computable sequence of well-founded trees $\left(T_{n}\right)_{n \in \mathbb{N}}$ with ranks at most $\alpha$ such that $X_{n}=\left[T_{n}\right]_{\Sigma}$. Idem with uniformly $\Pi_{\alpha}^{0}(E)$.

$3 G_{\delta}$ and $F_{\sigma}$ are the classes of intersections of uniformly $\Sigma_{1}^{0}(E)$ sequences (respectively unions of uniformly $\Pi_{1}^{0}(E)$ sequences).

\section{Remark 5.12.}

1 If $\leq$ is an order on $\mathbb{N}$ isomorphic to the ordinal $\alpha$ then the set of sequences $\left(n_{1}, \ldots, n_{k}\right)$ such that $n_{1} \geq n_{2} \ldots \geq n_{k}$ is a well-founded tree with rank $\alpha$ and computable rank order relation (i.e. it satisfies the above condition (a)).

2 The requirement that the rank order relation on $T$ is computable (in condition 1) allows to get the usual definition of $\Sigma_{\alpha}^{0}(E)$ for finite $\alpha$ 's. For instance, a subset $X$ of $E$ is $\Sigma_{2}^{0}(E)$ (respectively $\Sigma_{3}^{0}(E)$ ) if and only if there exists computably enumerable sets $A, B \subseteq \mathbb{N}^{2}$ (respectively $\left.A, B \subseteq \mathbb{N}^{3}\right)$ such that $X=\bigcup_{i \in \mathbb{N}}\left(\bigcup_{j:(i, j) \in A} O_{j}\right) \backslash\left(\bigcup_{j:(i, j) \in B} O_{j}\right)$ (respectively, $X=\bigcup_{i \in \mathbb{N}} \bigcap_{j \in \mathbb{N}}\left(E \backslash \bigcup_{k:(i, j, k) \in A} O_{k}\right) \cup\left(\bigcup_{k:(i, j, k) \in B} O_{k}\right)$ ).

Proposition 5.13. All assertions in Proposition 2.2 hold for the effective Borel classes with the proviso that $1 \leq \alpha<\omega_{1}^{C K}$ and countable unions (respectively intersections) are relative to uniformly $\Sigma_{\alpha}^{0}(E)$ (respectively $\Pi_{\alpha}^{0}(E)$ ) sequences of sets.

\subsection{Hausdorff Codes and the Effective Hausdorff Hierarchy}

The definition of the effective difference hierarchy for countably based spaces and their basic properties appeared previously in (Selivanov 2008).

Definition 5.14. Let $\left(E,\left(O_{n}\right)_{n \in \mathbb{N}}\right)$ be an effective topological space. 
1 A Hausdorff $\alpha$-code for a set $X$ in $\mathbf{D}_{\alpha}\left(\boldsymbol{\Sigma}_{\beta}^{0}(E)\right)$ is a triple $\left(\leq, P,\left(T_{n}\right)_{n \in \mathbb{N}}\right)$ such that

- $\leq$ is a well-order of type $\alpha$ on $\mathbb{N}$ or on a finite initial segment of $\mathbb{N}$,

$-P=\{n \in \operatorname{dom}(\leq) \mid \varphi(n) \sim \alpha\}$ where $\varphi$ is the unique isomorphism from $(\operatorname{dom}(\leq), \leq)$ onto the ordinal $\alpha$,

- $\left(T_{n}\right)_{n \in \mathbb{N}}$ is a family of well-founded trees with ranks at most $\beta$,

$-X=\cup_{p \in P}\left[T_{p}\right]_{\Sigma} \backslash \cup_{\varphi(q)<\varphi(p)}\left[T_{q}\right]_{\Sigma}$.

2 The effective Hausdorff classes $\mathrm{D}_{\alpha}\left(\Sigma_{\beta}^{0}(E)\right)$ are defined as follows: for $1 \leq \alpha<\omega_{1}^{C K}$, a set $X$ is in $\mathrm{D}_{\alpha}\left(\Sigma_{\beta}^{0}(E)\right)$ if and only if it admits an $\alpha$-code $\left(\leq, P,\left(T_{n}\right)_{n \in \mathbb{N}}\right)$ such that $\leq$ and $P$ are computable and the $T_{n}$ 's and the rank relations on the $T_{n}$ 's are uniformly computable, (i.e. $\left\{(n, s) \mid s \in T_{n}\right\}$ and $\left\{(n, s, t) \mid s, t \in T_{n}, \operatorname{rank}_{T_{n}}(s) \leq \operatorname{rank}_{T_{n}}(t)\right\}$ are computable).

The effective class $\mathrm{D}_{\alpha}\left(\Sigma_{1}^{0}(E)\right)$ is also denoted by $\mathrm{D}_{\alpha}(E)$.

Proposition 5.15. Propositions 2.6 2.9 and 2.10 hold with the effective Hausdorff classes and effectively continuous maps.

\subsection{Does Hausdorff's Theorem Fully Effectivize?}

Open Problem 5.16. Equality $\bigcup_{\alpha<\omega_{1}^{C}} \mathrm{D}_{\alpha}(E)=\Delta_{2}^{0}(E)$ holds in computable Polish spaces, (cf. Selivanov 2003, pages 76-79 for the Baire space). Is this also true for more general spaces including effective $\omega$-continuous domains endowed with the Scott topology?

Contrary to what was the case with the other results, the proof of Theorem 3.16 does not effectivize. The reason is that although the topological closure of a $\Delta_{2}^{0}$ set is closed, hence $\Pi_{1}^{0}$, it may not be $\Pi_{1}^{0}$. For instance, let $X$ be any countable $\Delta_{2}^{0}(\mathbb{N})$ subset of $\mathbb{N}$. In the real line, $X$ is $\Delta_{2}^{0}(\mathbb{R})$ and closed hence $\Pi_{1}^{0}(\mathbb{R})$, but $X$ is not $\Pi_{1}^{0}(\mathbb{R})$. Idem in the Baire space with the set $\left\{f \in \mathbb{N}^{\omega} \mid f(0) \in X\right\}$. This difficulty is mentioned in (Selivanov 2005), page 53, lines 6-7, with open sets: in the proof of his Theorem 3.10 page 50, open sets $A_{\beta}$ 's are defined using some $\Delta_{2}^{0}$ set $A$ : this is a stumbling block to get $\Sigma_{1}^{0}$ sets. For the Baire space $\mathbb{N}^{\omega}$, Selivanov (2003) uses a proof different from Hausdorff's original one. We adapt it to get the following weak effective version of Hausdorff's theorem.

Theorem 5.17. Let $\left(E,\left(O_{n}\right)_{n \in \mathbb{N}}, \ll\right)$ be an effective topological approximation space. Then $\left(F_{\sigma} \cap G_{\delta}\right)(E) \subseteq \bigcup_{\alpha<\omega_{1}^{C K}} \mathrm{D}_{\alpha}(E) \subseteq \Delta_{2}^{0}(D)$. In particular, if $\left(F_{\sigma} \cap G_{\delta}\right)(E)=\Delta_{2}^{0}(E)$ then the effective Hausdorff's theorem holds.

Proof. Without loss of generality, we suppose that the family $\left(O_{n}\right)_{n \in \mathbb{N}}$ is effectively closed under finite union: $O_{i_{1}} \cup \ldots \cup O_{i_{k}}=O_{\lambda\left(\left\{i_{1}, \ldots, i_{k}\right\}\right)}$ for some computable function $\lambda: \mathcal{P}_{<\omega}(\mathbb{N}) \backslash\{\varnothing\} \rightarrow \mathbb{N}$. Using Remark 5.12, for $\varepsilon=0,1$, let $\left(I_{n}^{\varepsilon}\right)_{n \in \mathbb{N}}$ be a family of subsets of $\mathbb{N}$ such that $\left\{(n, i) \mid i \in I_{n}^{\varepsilon}\right\}$ is computably enumerable and

$$
A=\bigcap_{n \in \mathbb{N}} \bigcup_{i \in I_{n}^{1}} O_{i} \quad, \quad E \backslash A=\bigcap_{n \in \mathbb{N}} \bigcup_{i \in I_{n}^{0}} O_{i} .
$$


We can suppose that the $I_{n}^{\varepsilon}$ 's are closed under the above function $\lambda$. Let $R$ be the computably enumerable set $R=\left\{(i, j) \mid O_{i} \ll O_{j}\right\}$. Let $R^{(t)}, I_{n}^{\varepsilon, t}$ be the finite parts of $R$ and $I_{n}^{t}$ obtained after $t$ steps of enumeration. Let $F_{\varepsilon}: \mathbb{N}^{2} \rightarrow \mathbb{N}$ be the function such that

$$
F_{\varepsilon}(m, t)=\max \left\{p \mid 0 \leq p \leq t \text { and } \forall q<p\left(m, \lambda\left(I_{q}^{\varepsilon, t}\right)\right) \in R^{(t)}\right\}
$$

In particular,

$$
O_{m} \subseteq \bigcap_{q<F_{\varepsilon}(m, t)} \bigcup_{i \in I_{q}^{\varepsilon, t}} O_{i} \subseteq \bigcap_{q<F_{\varepsilon}(m, t)} \bigcup_{i \in I_{q}^{\varepsilon}} O_{i} .
$$

We define a family $\mathcal{T}$ of finite sequences of integers in $\mathbb{N}$. The empty sequence is in $\mathcal{T}$. A sequence $\left(t_{0}, \ldots, t_{k}\right)$ is in $\mathcal{T}$ if and only if the following conditions are satisfied:

(a) $m_{0}<\ldots<m_{k}$ and $t_{0}<\ldots<t_{k}$,

(b) $\left(m_{\ell+1}, m_{\ell}\right) \in R^{\left(t_{\ell+1}\right)}$ for all $\ell<k$. In particular, this says that the sequence of subsets $\left(O_{m_{\ell}}\right)_{\ell=0, \ldots, k}$ is decreasing.

(c) For all $\ell \leq k, F_{0}\left(m_{\ell}, t_{\ell}\right) \neq F_{1}\left(m_{\ell}, t_{\ell}\right)$. For all $\ell<k$, if $F_{\varepsilon}\left(m_{\ell}, t_{\ell}\right)<F_{1-\varepsilon}\left(m_{\ell}, t_{\ell}\right)$ then $F_{1-\varepsilon}\left(m_{\ell+1}, t_{\ell+1}\right)<F_{\varepsilon}\left(m_{\ell+1}, t_{\ell+1}\right)$.

It is clear that, as a family of finite sequences, $\mathcal{T}$ is a tree and is computable.

Claim 1. The tree $\mathcal{T}$ is well-founded (i.e. it has no infinite branch).

Proof of Claim 1. Else consider an infinite branch $\left(m_{\ell}, t_{\ell}\right)_{\ell \in \mathbb{N}}$. Condition (3) of the Definition [5.11] and condition (b) of the definition of $\mathcal{T}$ imply that the set $\bigcap_{\ell \in \mathbb{N}} O_{m_{\ell}}$ contains at least one element $x$. Observe that condition (c) implies that $F_{\varepsilon}\left(m_{\ell}, t\right) \geq\lfloor\ell / 2\rfloor$. Using ( $\dagger$ ) for even and odd $n$ 's, we see that $x \in \bigcap_{q<\lfloor\ell / 2\rfloor} \bigcup_{i \in I_{q}^{\varepsilon}} O_{i}$ for all $\ell$ and for $\varepsilon=0,1$. Thus, $x$ is in both $A$ and $E \backslash A$, a contradiction. Let us say that a pair $(m, t) \in X$ has type $\varepsilon$ if $F_{\varepsilon}(m, t)>F_{1-\varepsilon}(m, t)$.

Claim 2. Suppose $x \in A$ and $(m, t) \in X$ has type 0 and $x \in O_{m}$. Then there exists $(p, u) \in X$ such that $(p, u)$ has type 1 and $x \in O_{p}$ and $(p, m) \in R, m<p$ and $t \leq u$. Switching types 0 and 1 , the same is true with $x \notin A$.

Proof of Claim 2. We treat the sole case $x \in A$, the other one being trivial modification. Since $x \notin E \backslash A$, condition $(*)$ insures that there exists $n$ such that $x \notin \bigcup_{i \in I_{n}^{0}} O_{i}$. Since $x \in A$, using again $(*)$, we see that $x \in \bigcap_{r \leq n} \cup_{i \in I_{r}^{1}} O_{i}$. Choose $i_{1}, \ldots, i_{n}, p$ such that $x \in O_{i_{r}}$ and $\left(i_{r}, p\right) \in R$ for all $r \leq n$. Let $u>t, n, i_{1}, \ldots, i_{n}$. Then $F_{0}(p, u)<n$ whereas $F_{1}(p, u) \geq n$ hence $(p, u)$ has type 1 and satisfies Claim 2 . We extend à la Kleene-Brouwer the computable well-founded reverse prefix partial ordering on $\mathcal{T}$ into a computable total well-ordering $\leq$ on $\mathcal{T}$ : let $\sigma, \tau \in \mathcal{T}$,

- If the sequences $\sigma$ and $\tau$ are prefix comparable, then we $\leq$-compare them relative to the reverse prefix partial order.

- If the sequences $\sigma$ and $\tau$ are not prefix comparable, then we compare the first elements on which they differ relative to the usual order on $\mathbb{N}$.

Using Ershov's trick, cf. Remark 5.10, we consider the set $\mathcal{S}=\mathcal{T} \times(\omega+2)$ well-ordered lexicographically using $\leq$ on $\mathcal{T}$ and the ordering on the ordinal $\omega+2=\{0,1,2, \ldots, \omega, \omega+1\}$. In other words, $\mathcal{S}$ is obtained from $\mathcal{T}$ by replacing each element of $\mathcal{T}$ by a chain of $\omega+2$ copies of that element. We also denote by $\leq$ the well-ordering on $\mathcal{S}$. The following Claim is straightforward. 
Claim 3. $(\mathcal{S}, \leq)$ is a computable well-ordering and its order type is an even ordinal. Moreover, the parity of the rank relative to $\leq$ of an element $(\sigma, \gamma) \in \mathcal{S}$ is equal to the parity of the ordinal $\gamma$. In particular, this parity function is computable. Attach to any element $(\sigma, \theta) \in \mathcal{S}$ an open set $U(\sigma, \theta)$ as follows: If $\sigma$ is the empty sequence then $U(\sigma, \theta)=\varnothing$. Else, if the last pair $\left(m_{k}, t_{k}\right)$ of $\sigma$ has type $\varepsilon \in\{0,1\}$ then

$$
\forall n<\omega U(\sigma, n)=\varnothing \quad, \quad U(\sigma, \omega+\varepsilon)=O_{m_{k}} \quad, \quad U(\sigma, \omega+(1-\varepsilon))=\varnothing .
$$

If $\alpha$ is the rank of the element $(\sigma, \theta)$ in $T$ then let $A_{\alpha}=U(\sigma, \theta)$. The family $\left(A_{\alpha}\right)_{\alpha<\xi}$ is an effective family of open sets in the sense of Definition [5.14, Let $\xi$ be the (limit) ordinal to which $(\mathcal{S}, \leq)$ is isomorphic. The following Claim finishes the proof.

Claim 4. $D_{\xi}\left(\left(A_{\alpha}\right)_{\alpha<\xi}\right)=A$.

Proof of Claim 4. Suppose $x \in A$. By Claim 2 there exists a pair $(m, t)$ such that the one element sequence $\tau=((m, t))$ is in $\mathcal{T}$, has type 1 and $x \in U(\tau, \omega+1)$. Thus, we can consider the least ordinal $\alpha$ which is the rank of some $(\sigma, \gamma) \in \mathcal{S}$ such that $x \in U(\sigma, \gamma)$. First, we show that $\alpha$ is odd. Since $U(\sigma, \gamma)$ is not empty, its rank is of the form $(\omega+2) \delta+\omega+\varepsilon$ where $\varepsilon$ is the type of $\sigma$. If $\varepsilon=0$ then Claim 2 would allow to extend $\sigma$ to $\sigma(p, u) \in \mathcal{T}$ such that $x \in O_{p}$ and $\sigma(p, u)$ has type 1 . Since $\sigma(p, u)$ extends $\sigma$ it has lesser rank in $\mathcal{T}$. Thus, $x \in U(\sigma(p, u), \delta)$ where $(\sigma(p, u), \delta)$ has lesser rank than $(\sigma, \gamma)$, a contradiction. By definition of $\alpha$, we have $A_{\alpha}=U(\sigma, \gamma)$ hence $x \in A_{\alpha}$. The choice of $\alpha$ insures that $x$ is in no $A_{\beta}$ for $\beta<\alpha$. Thus, $x \in A_{\alpha} \backslash \bigcup_{\beta<\alpha} A_{\beta}$ with $\alpha$ odd. This shows that $x \in \mathrm{D}\left(\left(A_{\mu}\right)_{\mu<\xi}\right)$. A similar argument shows that if $x \notin A$ then $x \in A_{\alpha} \backslash \bigcup_{\beta<\alpha} A_{\beta}$ with $\alpha$ even hence $x \notin \mathrm{D}\left(\left(A_{\mu}\right)_{\mu<\xi}\right)$. Summing up, we see that $A=\mathrm{D}\left(\left(A_{\mu}\right)_{\mu<\xi}\right)$.

Corollary 5.18. In every effective $\omega$-continuous domain $D$,

$$
G_{\delta}(D) \cap F_{\sigma}(D) \subseteq \bigcup_{\alpha<\omega_{1}} \mathrm{D}_{\alpha}(D) \subseteq \Delta_{2}^{0}(D) .
$$

\section{References}

Samson Abramsky and Achim Jung. Domain theory. In Handbook of Logic in Computer Science, vol. III. Abramsky, Gabbay \& Maibaum, editors. Oxford University Press, 1994

Verónica Becher and Serge Grigorieff. Wadge hardness in Scott spaces and its effectivization. This volume, 2012.

Harold Bennett and David Lutzer. Strong completeness properties in topology. Questions and Answers in General Topology, 27(2009), 107-12, 2009.

Matthew de Brecht. Quasi-Polish spaces. arXiv:1108.1445v1, 40 pages, 2011.

Gustave Choquet. Lectures on analysis. Vol. I: Integration and topological vector spaces. Benjamin, 1969.

G. Debs. An example of an $\alpha$-favourable topological space with no $\alpha$-winning tactic. Séminaire d'Initiation a l'Analyse (Choquet-Rogalski-Saint Raymond). 1984.

G. Debs. Stratégies gagnantes dans certains jeux topologiques. Fundamenta Mathematicae, 126:93-105, 1985.

Patrick Dehornoy. Turing complexity of the ordinals. Information Processing Letters, 23(4):167$170,1986$.

François G. Dorais and Carl Mummert. Stationary and convergent strategies in Choquet games. Fundamenta Mathematicae, 209:59-79, 2010. 
Abbas Edalat. Domains for computation in mathematics, physics and exact real aritmetic. Bulletin of Symbolic Logic, 3(4):401-452, 1997.

Yuri Ershov. On a hierarchy of sets II. Algebra and Logic, 7(4):15-47, 1968.

Fred Galvin \& RatislavTelgárky. Stationary strategies in topological games. Topology and its applications, 22:51-69, 1986.

G. Gierz, K. H. .Hofmann, K. Keimel, J. D. Lawson, M. Mislove and D. S. Scott. Continuous Lattices and Domains. Cambridge University Press, 2003.

Serge Grigorieff. Every recursive linear ordering has an isomorphic copy in DTIME$\operatorname{SPACE}(n, \log (n))$. Journal of Symbolic Logic, 55(1):260-276, 1990.

Peter Hertling. Unstetigkeitgrade con Funktionen in der effektiven Analysis. PhD thesis, FernUniversity in Hagen, 1996.

Peter Hertling. Topological complexity with continuous operations. Journal of Complexity, 12(4):315-338, 1996.

Alexander S. Kechris. Classical Descriptive Set Theory. Springer, 1995.

Hans-Peter Künzi. On strongly quasi-metrizable spaces. Archiv der Mathematik, 41(1):57-63, 1983.

Kazimierz Kuratowski. Topology. Volume I. Academic Press, 1966.

David Marker. Descriptive set theory. Lecture Notes. On Marker's home page, 2002.

Yiannis Moschovakis. Descriptive set theory, volume 155. American Mathematical Society. First edition 1979, second edition 2009.

J.C. Oxtoby. The Banach-Mazur game and Banach Category Theorem. In Contributions to the theory of games, Vol. III, Annals of Math. Studies 39:159-163, 1957.

W.W. Schmidt. On badly approximable numbers and certain games. Transactions Amer. Math. Soc., 123:178-199, 1966.

Victor L. Selivanov. Wadge degrees of $\omega$-languages of deterministic Turing machines. Theoretical Informatics and Applications, 37(1):67-83, 2003. Extended abstract in STACS 2003 Proceedings, Lecture Notes in Computer Science 2607:97-108, 2003.

Victor L. Selivanov. Hierarchies in $\varphi$-spaces and applications. Mathematical Logic Quaterly, 51(1):45-61, 2005.

Victor L. Selivanov. Towards a descriptive set theory for domain-like structures. Theoretical Computer Science, 365(3):258-282, 2006.

Victor L. Selivanov. On the difference hierarchy in countably based $T_{0}$-spaces. Electronic Notes in Theoretical Computer Science, 221:257-269, 2008.

Clifford Spector. Recursive well-orderings. Journal of Symbolic Logic, 20(2):151-163, 1955.

A. Tang. Wadge reducibility and hausdorff difference hierarchy in $P \omega$. Lectures Notes in Mathematics 871:360-371, 1981.

Klaus Weihrauch. Computable analysis. An introduction. Springer, 2000. 